\title{
How do autoimmune diseases cluster in families? A systematic review and meta-analysis
}

Jorge Cárdenas-Roldán, Adriana Rojas-Villarraga and Juan-Manuel Anaya*

\begin{abstract}
Background: A primary characteristic of complex genetic diseases is that affected individuals tend to cluster in families (that is, familial aggregation). Aggregation of the same autoimmune condition, also referred to as familial autoimmune disease, has been extensively evaluated. However, aggregation of diverse autoimmune diseases, also known as familial autoimmunity, has been overlooked. Therefore, a systematic review and meta-analysis were performed aimed at gathering evidence about this topic.

Methods: Familial autoimmunity was investigated in five major autoimmune diseases, namely, rheumatoid arthritis, systemic lupus erythematosus, autoimmune thyroid disease, multiple sclerosis and type 1 diabetes mellitus. Preferred Reporting Items for Systematic Reviews and Meta-Analysis (PRISMA) guidelines were followed. Articles were searched in Pubmed and Embase databases.

Results: Out of a total of 61 articles, 44 were selected for final analysis. Familial autoimmunity was found in all the autoimmune diseases investigated. Aggregation of autoimmune thyroid disease, followed by systemic lupus erythematosus and rheumatoid arthritis, was the most encountered.
\end{abstract}

Conclusions: Familial autoimmunity is a frequently seen condition. Further study of familial autoimmunity will help to decipher the common mechanisms of autoimmunity.

Keywords: Autoimmune diseases, familial autoimmunity, aggregation, genetic epidemiology, autoimmune tautology

\section{Background}

Autoimmune diseases (ADs) are chronic conditions initiated by the loss of immunological tolerance to selfantigens; they represent a heterogeneous group of disorders that afflict specific target organs or multiple organ systems [1]. The chronic nature of these diseases places a significant burden on the utilization of medical care, increases direct and indirect economic costs, and diminishes quality of life. The estimated incidence of ADs is approximately 80 per 100,000 person years and their prevalence could be well beyond 3\% of the population [2]. Most of the ADs asymmetrically affect middle-aged women and are among the leading causes of death for this group of patients. Although the frequency of ADs varies

\footnotetext{
* Correspondence: juan.anaya@urosario.edu.co
Center for Autoimmune Diseases Research (CREA), School of Medicine and

* Correspondence: juan.anaya@urosario.edu.co
Center for Autoimmune Diseases Research (CREA), School of Medicine and Health Sciences, Universidad del Rosario, Carrera 24 \#63-C-69, Bogota, Colombia
}

(c) 2013 Cárdenas-Roldán et al; licensee BioMed Central Ltd. This is an Open Access article distributed under the terms of the Creative Commons Attribution License (http://creativecommons.org/licenses/by/2.0), which permits unrestricted use, distribution, and reproduction in any medium, provided the original work is properly cited. some ADs, ADs share several clinical signs and symptoms (that is, subphenotypes), physiopathological mechanisms, and genetic factors. These shared characteristics have been grouped under the term autoimmune tautology [5-10]. In clinical practice two conditions support this theory, namely, polyautoimmunity and familial autoimmunity, both of which are considered as being part of the "kaleidoscope of autoimmunity' [11-14]. Whereas polyautoimmunity is the presence of two or more ADs in a single patient, familial autoimmunity occurs when relatives from a nuclear family present diverse ADs [9] (Figure 1). These conditions indicate that similar genetic, epigenetic, and environmental factors influence the development of ADs [7]. The best examples of polyautoimmunity are the multiple autoimmune syndrome (MAS), which occurs when a patient has three or more ADs $[15,16]$, and the 


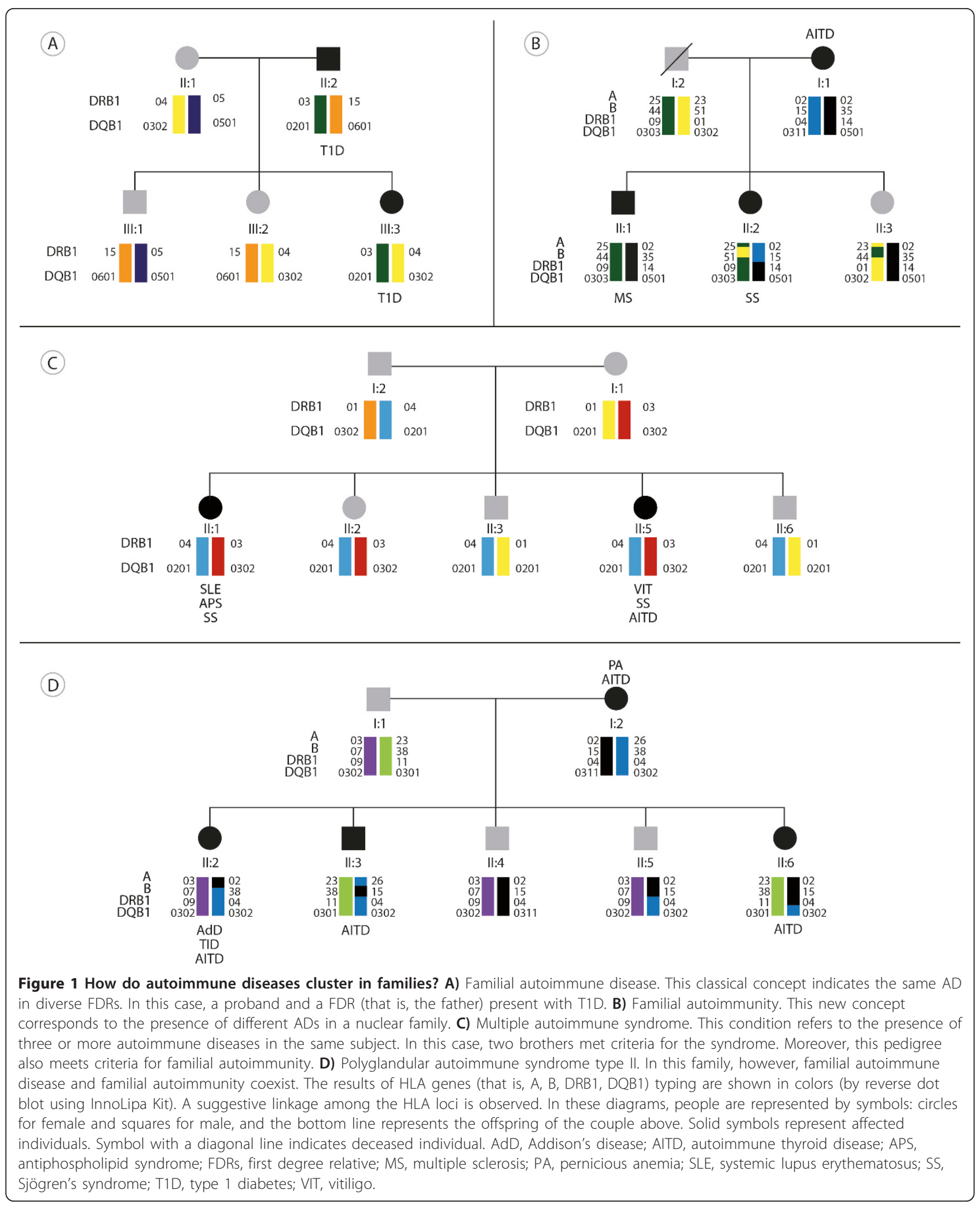


polyglandular autoimmune syndromes type II, III and IV [17], which are in fact MAS.

ADs do not begin at the moment they become clinically apparent but several years before. This implies that there is a chance to predict autoimmunity. Over the years, several risk factors have been associated with the onset of ADs. Among these the most widely studied are female gender [18], specific alleles at HLA and nonHLA loci $[2,19]$ and some environmental agents $[20,21]$. In addition, the presence of auto antibodies may also predict specific clinical manifestations, disease severity and disease progression [22-27]. As reviewed by Tobon et al. [5] many auto antibodies have a predictive ability and they can be serologically evaluated long before the appearance of clinical disease. Thus, identification of these markers as well as a family history of autoimmunity and evaluation of their predictive value could be useful for personalized medicine.

A primary characteristic of complex diseases is that they are likely to aggregate in families (that is, familial aggregation, also referred to as recurrence risk or lambda, $\lambda$ ). The aggregation of a phenotype is observed when a disease occurs at a higher frequency in the relatives of an affected individual as compared with the frequency observed in the general population. Values of $\lambda>1.0$ indicate aggregation [9]. Aggregation of the same autoimmune condition, also referred to as familial autoimmune disease, has been extensively evaluated. However, aggregation of diverse autoimmune diseases, also known as familial autoimmunity, has been overlooked (Figure 1). Therefore, a systematic review and meta-analysis were performed aimed at gathering evidence about this topic.

\section{Methods}

\section{Systematic review}

A literature search was done even though 'familial autoimmunity' is not a Medical Subject Headings (MeSH) term. Nevertheless, the search was done in the electronic databases Medline and Embase, and included articles, from 1966 for the former and 1980 for the latter, up to June 2012. The search strategy was limited to humans and included the words '(familial OR clustering OR aggregation)' AND 'autoimmunity followed by each of the diseases we have focused on: 'multiple sclerosis,' 'diabetes mellitus, Type 1,' 'arthritis, rheumatoid' and 'lupus erythematosus, systemic' using $\mathrm{MeSH}$ terms and key words for 'autoimmune thyroid disease'. In order not to miss potentially eligible studies we used wild cards for the words familial, clustering and aggregation in the following manner: famil*, aggrega* and cluster*. No language restrictions were used. Articles were included if they fulfilled the following conditions: ADs diagnosis was carried out according to international criteria or through international classification of diseases, articles were published as full articles and, as mentioned earlier, if ADs in first degree relatives (FDRs) were different than in the proband. Studies were excluded if they only referred to autoantibody prevalence, if a clear cut distinction between diseases was not possible, if it was not possible to distinguish between probands and FDRs, if the studies were case reports, and if they dealt with a single family. Unpublished data were also excluded. Eligibility assessment was done by a primary reviewer who screened all titles and abstracts of publications. Retrieved articles were rejected if eligibility criteria were not met and a secondary reviewer was consulted in cases in which eligibility criteria were unclear. References from the articles that seemed to be relevant for our review were hand-searched. All articles were assessed according to the Oxford Centre for Evidencebased Medicine 2011 Levels of Evidence [28]. The search returned articles in which familial autoimmunity was assessed in other ADs and they were included. From each study we extracted data including total number of FDRs, numbers of FDRs affected, prevalence of ADs and, where possible, extraction of crude and adjusted measures of association, that is, odds ratio (OR) or risk ratio (RR). With the prevalences extracted, aggregation for different ADs across the five index diseases mentioned earlier was calculated by dividing the prevalence of a given AD in FDRs by the prevalence in the general population $\left(\lambda_{\text {relatives }}\right)$. We extracted data on prevalences from five reports [2,29-32]. Inclusion criteria for the meta-analyses were applied to publications that provided epidemiological data on risk factors, RR and OR with confidence intervals $(\mathrm{CI})$, or that provided information that allowed us to calculate these data. If the study did not report the number of subjects in each group, either the RR or the OR with the CI, must have been reported in order for them to be included in the meta-analyses calculations.

In order to study aggregation, we determined worldwide prevalences of ADs from five studies mentioned earlier [2,29-32]. If a range was reported, we arbitrarily calculated the mean.

\section{Meta-analyses}

Data were analyzed using the Comprehensive MetaAnalysis Version 2 program (Biostat, Englewood, NJ, 2004). Calculations were carried out for the whole group of articles depending on the binary data available for any AD: number of subjects and risk data (OR and RR with the corresponding 95\% CI). Effect size was calculated based on studies that reported an OR with its respective $95 \% \mathrm{CI}$ and from raw data given by case-control and cohort studies. If raw data from cohort studies were available, a second effect size was calculated with studies that only showed the RR and the respective 95\% 
CI. Different study designs were used to compute the same effect size since the effect size had the same meaning in all studies and was comparable in relevant aspects. In order to perform the analyses, the association measures were transformed to log values, and then the results were converted back to ratio values for presentation. This approach prevented the omission of studies that used an alternative measure. Two types of metaanalyses were done in order to analyze autoimmunity as a trait. First, a given AD in FDRs was analyzed through all the studies regardless of the AD of the proband. The second type of meta-analysis analyzed ADs in FDRs through all the studies from a specific AD present in the proband.

Additional meta-analyses were done for studies with complex data structures and non-cumulative results as the information for the different effects was not totally independent. This is the case for studies reporting multiple independent subgroups, that is, aggregation for son and daughter separately, within a study. A flow diagram of the current study is shown in Figure 2.

ORs were grouped by weighing individual ORs by the inverse of their variance. For each analysis, the final effect OR and 95\% CI were obtained by means of the random effect model, which was preferred because it accepts distributions of true effect sizes rather than one true effect and assigns a more balanced weight to each study. It was also used because all the studies were considered to be unequal in terms of specific ADs.

Heterogeneity was calculated by means of Higgins's $\left(I^{2}\right)$ tests. The variance between studies was estimated by the DerSimonian and Laird method. The $I^{2}$ test showed the proportion of observed dispersion that was real rather than spurious and was expressed as a ratio ranging from $0 \%$ to $100 \% . I^{2}$ values of $25 \%, 50 \%$, and $75 \%$ were qualitatively classified as low, moderate, and high, respectively. Publication bias was determined using Funnel plots and Egger's regression asymmetry tests.

\section{Results}

\section{Studies retrieved}

After discarding duplicates, the search in both databases retrieved 2,552 articles. In a first assessment we considered 61 articles to be eligible. In a second screening 17 of these articles were not eligible due to reporting inconsistencies, such as not distinguishing between probands and FDRs. As we did not identify other articles from the reference lists, only 44 articles met eligibility criteria [32-75]. Figure 3 and Table 1 summarize the search results. Although ankylosing spondylitis (AS) is considered an auto-inflammatory more than autoimmune disease [76], it was included in the results since it was found to aggregate in families. Most of the studies found lacked controls and had a small sample size, which is reflected in low grading according to the 2011 levels of evidence from the Oxford Centre for Evidencebased Medicine [28]. Detailed information is shown in Table 2.

\section{Autoimmune thyroid disease}

Three articles assessed familial autoimmunity for autoimmune thyroid disease (AITD) [33-35]. AITD encompasses Graves' disease as well as Hashimoto's thyroiditis with the latter being the most common cause of acquired hypothyroidism [77]. Moreover, AITD is the most common AD [78]. Various studies have shown that AITD coexists with other ADs in the same subject $[79,80]$ and it has also been shown that there is familial clustering of AITD in FDRs, particularly in female relatives [81]. Boelaert et al. [33] described familial autoimmunity among probands with Hashimoto's thyroiditis or Graves' disease. Both ADs were significantly associated with the presence of type 1 diabetes mellitus (T1D), rheumatoid arthritis (RA), pernicious anemia (PA), systemic lupus erythematosus (SLE), celiac disease (CD), vitiligo (VIT) and multiple sclerosis (MS). Only Graves' disease was associated with Addison's disease (AdD) and inflammatory bowel disease (IBD). Compared with the general population, familial autoimmunity in Graves' disease probands disclosed PA as the strongest association (RR: 14.1 ; 95\% CI: 11.48 to 17.03 ), followed by RA (RR: 13.5 ; $95 \%$ CI: 12.32 to 14.86$)$.

Hemminki et al. [34] assessed familial autoimmunity only in probands with Graves' disease from Sweden. To calculate familial risk within a large community based cohort they calculated standardized incidence ratios (SIR) as the ratio between the observed and the expected frequency for each disease. A value over one indicates an increased frequency of what is expected whereas a value below one indicates a decreased frequency. The analysis was stratified according to the FDR involved. For a single parent affected, Hashimoto's disease, PA, and RA were the only diseases significantly associated, having a SIR of 2.04, 1.82 and 1.48, respectively, thus showing an increased frequency of what is expected. Significant associations for singleton siblings were found for T1D, discoid lupus and localized scleroderma, having a SIR of 2.14, 6.03 and 6.62, respectively. If a parent and a sibling were affected with the same $\mathrm{AD}$, the significant association was between Hashimoto's disease with a SIR of 37.41 and SLE with a SIR of 14.33 [34].

\section{Type 1 diabetes mellitus}

The search returned seven articles about T1D probands [35-41]. AITD was responsible for the familial autoimmunity found in most of the articles $[36,40]$, even when compared to control subjects [37]. Wagner et al. [38] 


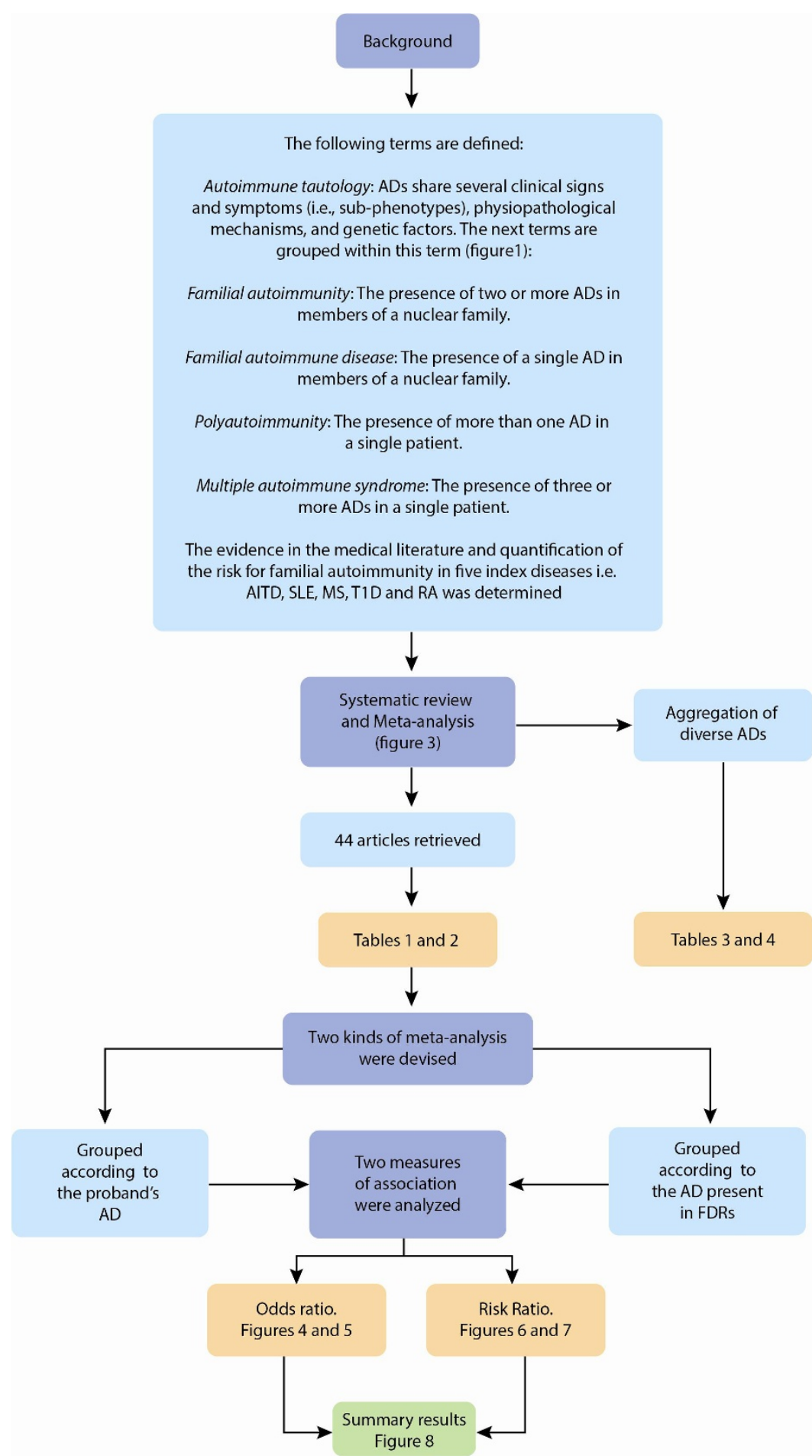

Figure 2 Flow diagram of current study. AITD, autoimmune thyroid disease; MS, multiple sclerosis; RA, rheumatoid arthritis; SLE, systemic lupus erythematosus; T1D, type 1 diabetes.

replicated the results but also described the presence of $\mathrm{CD}$, psoriasis (PSO), and VIT.

Hemminki et al. [39] also reported familial autoimmunity in probands with T1D. When a parent had AdD, the SIR for T1D in offspring was 2.41. It was 2.73 for CD, 1.83 for Graves' disease, 2.13 for Hashimoto's thyroiditis, 3.09 for PA, 3.63 for primary biliary cirrhosis (PBC), 2.12 for RA, 1.62 for SLE, 1.23 for ulcerative 


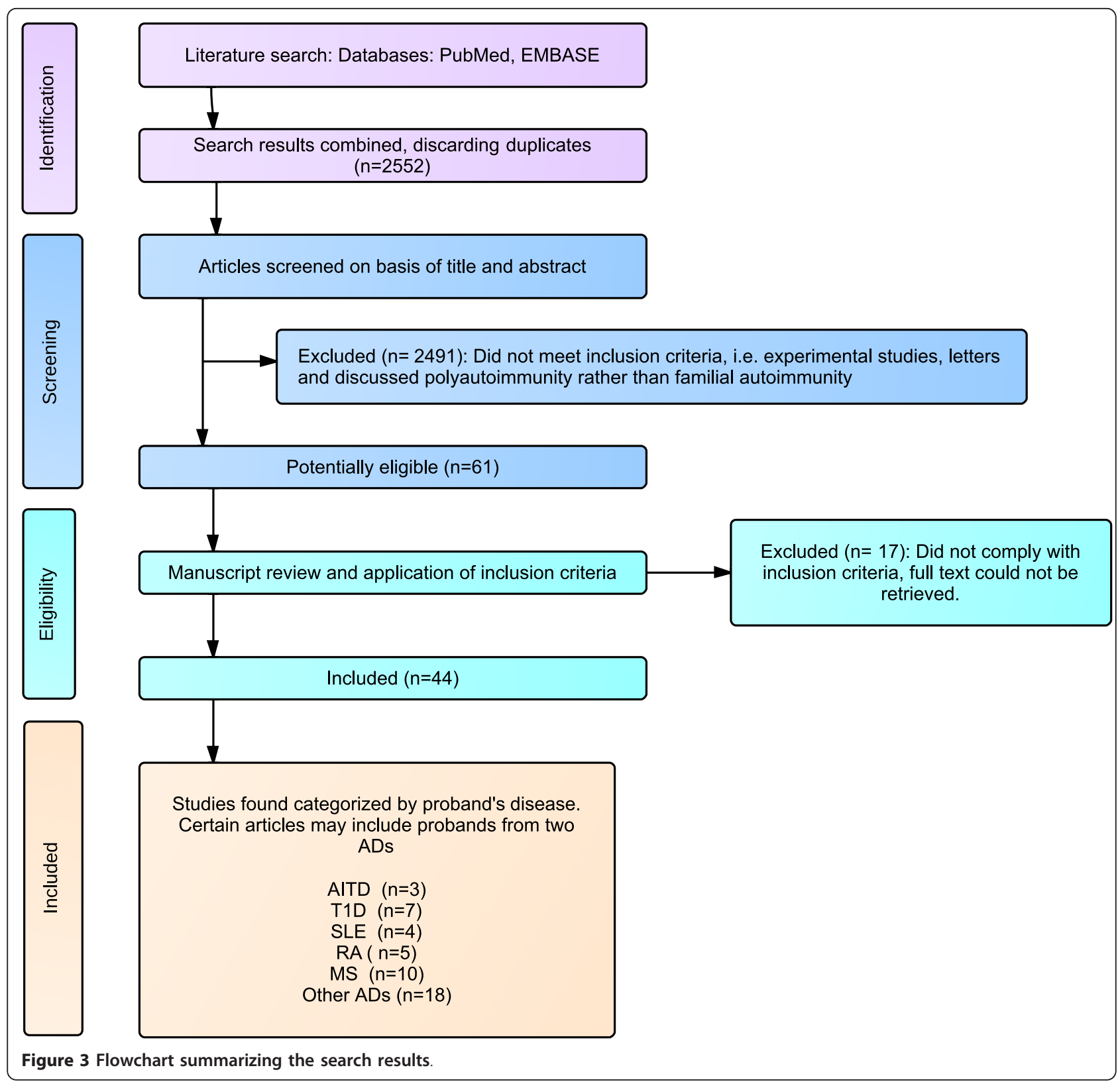

colitis (UC), and 1.23 for Wegener's granulomatosis (WG). Only the presence of AdD, CD or Graves' disease in singleton siblings was associated with T1D in probands. Likewise, when a parent and sibling had RA, the SIR for T1D was 5.34 [39].

\section{Systemic lupus erythematosus}

Four articles assessed familial autoimmunity in SLE probands. Alarcon-Segovia et al. evaluated familial aggregation in the "Grupo Latinoamericano de Estudio de Lupus' (GLADEL) [42]. They found that among all family members who had any AD, $6.7 \%$ had RA, $2 \%$
AITD and other ADs at a lesser frequency. In FDR ( $\mathrm{n}=$ $114)$ with ADs, $28 \%(\mathrm{n}=32)$ had RA and $16 \%(\mathrm{n}=32)$ had AITD [42]. Likewise, an increased frequency of familial autoimmunity was found in SLE probands compared with population prevalence. Priori et al. [43] found an OR of 4.6 (95\% CI 1.94 to 11.1$)$ in a multivariate analysis of familial autoimmunity in FDR of SLE patients. They reported AITD as the most frequent disease with eight cases, followed by RA with five cases, VIT with three cases and T1D with two cases. PSO frequency was higher among non-autoimmune controls. Sjögren's syndrome (SS) as well as AITD were described 
Table 1 Significant associations of autoimmune diseases in first degree relatives.

\begin{tabular}{|c|c|c|c|}
\hline Proband's disease & Author & Disease in first degree relatives & References \\
\hline \multirow[t]{4}{*}{ Autoimmune thyroid disease } & Boelaert et al. & AdD, CD, IBD, MG, MS, PA, RA, SLE, T1D, VIT & [33] \\
\hline & Hemminki et al. & In parent: AdD, IIM, MG, PA, RA, T1D, UC & {$[34]$} \\
\hline & & In sibling: Discoid lupus erythematosus, localized SSc, T1D & \\
\hline & & In parent and sibling: SLE & \\
\hline \multirow[t]{7}{*}{ Type 1 diabetes } & Bottazo et al. & AITD & {$[36]$} \\
\hline & Anaya et al. & AITD & [37] \\
\hline & Wagner et al. & AITD,CD, PSO, RA, VIT & [38] \\
\hline & Hemminki et al. & In parent: $A d D, A I T D, A S, C D, P A, P B C, R A, S L E, U C, W G$ & [39] \\
\hline & & In sibling: AdD, AITD, CD & \\
\hline & & In parent and sibling: RA & \\
\hline & Lebenthal et al. & AITD, CD & [41] \\
\hline \multirow[t]{4}{*}{ Systemic lupus erythematosus } & Criswell et al. & AITD & [35] \\
\hline & Alarcon-Segovia et al. & AITD, IIM, RA, SSC & {$[42]$} \\
\hline & Priori et al. & AITD, RA, T1D, VIT & {$[43]$} \\
\hline & Corporaal et al. & $\mathrm{MS}, \mathrm{RA}$ & [44] \\
\hline \multirow[t]{4}{*}{ Rheumatoid arthritis } & Lin et al. & AITD, T1D, & {$[45]$} \\
\hline & Thomas et al. & $\mathrm{T} 1 \mathrm{D}$ & {$[46]$} \\
\hline & Hemminki et al. & $\begin{array}{l}\text { In parent: AITD, AS, Localized SSC, PA, PSO, SLE, SS, SSC, WG } \\
\text { In sibling: PSO, SLE }\end{array}$ & [47] \\
\hline & Jawaheer et al. & AITD & {$[48]$} \\
\hline \multirow[t]{8}{*}{ Multiple sclerosis } & Barcellos et al. & AITD, PSO, RA, T1D & {$[32]$} \\
\hline & Criswell et al. & PSO & {$[35]$} \\
\hline & Broadley et al. & AITD & [49] \\
\hline & Deretzi et al. & AITD, IBD, PSO, T1D & {$[50]$} \\
\hline & Heinzlef et al. & AITD, RA, T1D, VIT & [51] \\
\hline & Henderson et al. & AS & {$[52]$} \\
\hline & Marrosu et al. & T1D & {$[53]$} \\
\hline & Nielsen et al. & $\mathrm{AdD}, \mathrm{CrD}, \mathrm{PAN}$ & {$[55]$} \\
\hline \multirow[t]{3}{*}{ Systemic sclerosis } & Arora-Singh et al. & AITD, RA, SLE, & {$[57]$} \\
\hline & Hudson et al. & AITD, PBC, RA, SLE, SS & {$[58]$} \\
\hline & Koumakis et al. & AITD, RA, SLE, SS & {$[59]$} \\
\hline \multirow[t]{2}{*}{ Sjögren's syndrome } & Reveille et al. & AITD, MS, SLE, SSC & [61] \\
\hline & Anaya et al. & AITD, RA, SLE & {$[62]$} \\
\hline Inflammatory bowel disease & Criswell et al. & MS & {$[35]$} \\
\hline \multirow[t]{3}{*}{ Ulcerative colitis } & Hemminki et al. & In parent: AITD, AS, CrD, MS, PA, PAN, PSO, RA, SLE, T1D & [63] \\
\hline & & In sibling: AS, CrD & \\
\hline & & In parent and sibling: CrD, PSO & \\
\hline \multirow[t]{4}{*}{ Crohn's disease } & Hemminki et al. & In parent: AS, PSO, UC & [63] \\
\hline & & In sibling: AS, UC & \\
\hline & & In parent and sibling: RA, UC & \\
\hline & & In twins: UC & \\
\hline \multirow[t]{3}{*}{ Vitiligo } & Alkhateeb et al. & AdD, AITD, MG, PA, SLE, SSC & [64] \\
\hline & Laberge et al. & AdD, AITD, PA, PSO, RA & [65] \\
\hline & Zhang et al. & $\mathrm{AA}, \mathrm{PSO}, \mathrm{RA}$ & [66] \\
\hline \multirow[t]{2}{*}{ Juvenile rheumatoid arthritis } & Prahalad et al. & AITD & [67] \\
\hline & Huang et al. & AITD, AS, PSO, SLE & [68] \\
\hline Juvenile lupus erythematosus & Huang et al. & AITD, AS, MG & [68] \\
\hline Inflammatory idiopathic myositis & Ginn et al. & AITD, PA, PSO, RA, SS, T1D & [70] \\
\hline Celiac disease & Petaros et al. & AITD, PSO, T1D & {$[72]$} \\
\hline
\end{tabular}


Table 1 Significant associations of autoimmune diseases in first degree relatives. (Continued)

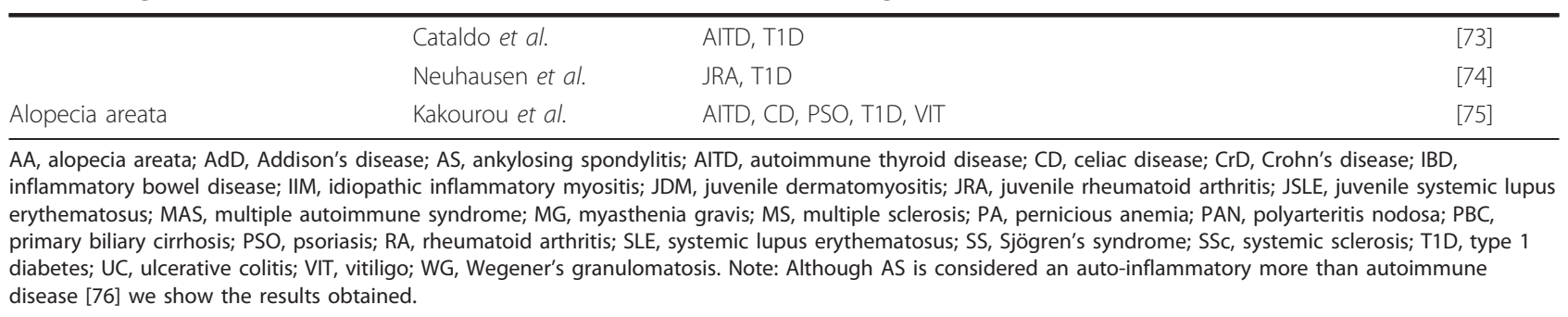

by Scofield et al. [82] while Corporaal et al. [44] found clustering of MS and RA.

\section{Rheumatoid arthritis}

In RA, familial autoimmunity was ascertained in five articles, all of which linked AITD or T1D to AR. Lin et al. [45] showed an association with AITD in $7.8 \%$ of the probands and T1D in $2.8 \%$. Thomas et al. [46] also reported $\mathrm{T} 1 \mathrm{D}$ as the disease responsible for familial autoimmunity. In another study, Taneja et al. [83] stated that SLE, T1D, AITD, SS, PSO and systemic sclerosis (SSc) were found in families with RA. However, they included probands within this description, thus assessing and combining polyautoimmunity or MAS with familial autoimmunity. Walker et al. [84] found an excess risk for AITD in RA multicase families compared with the general population. However, this significance was lost when RA sufferers were withdrawn from the analysis. Jawaheer et al. [48] found the presence of AITD and other ADs in siblings but, compared with siblings of non-RA probands, the difference was not significant.

Hemminki et al. [47] also reported familial autoimmunity in probands with RA. Just as described above, when a parent had AS, the SIR for RA in offspring was 2.96 . It was 2.25 for SS, 2.13 for SLE, 1.65 for SSc, 1.54 for AITD, 1.53 for PA, 1.36 for PSO and 1.34 for WG. When singleton siblings had PSO, the SIR for RA of the proband was 2.01 and 2.77 for SLE.

\section{Multiple sclerosis}

In our search, MS was the AD with the most articles assessing familial autoimmunity with 10 articles found $[32,35,49-56]$. Some studies suggest that FDRs and other relatives of probands with MS could be at greater risk of ADs other than MS [32,35,49-55,57] while the studies done by Ramagopalan et al. [54] and Midgard et al. [85] do not support these findings. Although Annunziata et al. [86] found an association between MS and other ADs in first and second degree relatives, the results were not significant when compared to non-AD controls. Conversely, Alonso et al. [87] and Magaña et al. [88] found a significant association between MS and other ADs in relatives of any degree.
Using 265 families from the Multiple Autoimmune Disease Genetics Consortium (MADGC), Criswell et al. [35] compared the frequency of ADs in siblings of multiplex families stratified by seven ADs: AITD, RA, MS, SLE, T1D, IBD and PSO. These diseases were pre-specified given a variety of considerations. There was no evidence of familial autoimmunity except in the case of IBD patients in whose families MS was observed among FDRs (OR: $8.1 ; 95 \%$ CI: 1.77 to $37.0 ; P$ value $=0.018$ ). However, selection bias was present as families selected for inclusion were not recruited in the same manner [35].

\section{Meta-analyses}

For the first effect size, OR, 13 meta-analyses were developed. Ten analyzed the proportion of a specific AD in FDRs independent of the AD present in the proband. Of these, three showed significant association: AITD, T1D and IBD. Three included an independent AD in FDRs in a specific AD of the proband, two of them showed significant associations: RA and MS. Figures 4 and 5 show the forest plots corresponding to six metaanalyses.

A second effect size was calculated based on data from studies showing RR data. Twenty eight meta-analyses were developed. Twenty three analyzed the proportion of a specific AD in the FDR through all the studies independent of any AD of the proband. Of these, nineteen showed significant association, the most relevant results being related to VIT, PA, RA and T1D. Additional results are shown in Additional file 1. Through all the studies, four additional analyses performed included any AD present in FDRs. All these analysis disclosed significant results. The ADs in the proband were AITD, MS, RA and T1D (Figures 6 and 7).

Evidence of significant publication bias was identified using the Egger test ( $P$-value 2 -tailed: $<0.05)$ for two meta-analyses which included studies that reported OR with its respective 95\% CI (T1D in FDR ( $P$-value 2-tailed: 0.047$)$ and MS in probands ( $P$-value 2 -tailed: 0.007)). One meta-analysis that reported RR data showed publication bias by the Egger test (AITD in probands ( $P$-value 2 -tailed: 0.008$)$ ) (Figure 6A). The 
Table 2 Characteristics of the studies included.

\begin{tabular}{|c|c|c|c|c|c|c|c|c|c|c|c|}
\hline $\begin{array}{l}\text { Disease in } \\
\text { probands }\end{array}$ & $\begin{array}{c}\text { Author and } \\
\text { year }\end{array}$ & Country & $\begin{array}{l}\text { Study } \\
\text { type }\end{array}$ & $\begin{array}{c}\text { Total } \\
\text { number of } \\
\text { probands }\end{array}$ & $\begin{array}{l}\text { Affected } \\
\text { FDRs in } \\
\text { probands }\end{array}$ & $\begin{array}{l}\text { Total } \\
\text { number } \\
\text { FDRs }\end{array}$ & $\begin{array}{l}\text { Total } \\
\text { controls }\end{array}$ & $\begin{array}{l}\text { Affected } \\
\text { FDRs in } \\
\text { controls }\end{array}$ & $\begin{array}{l}\text { Total } \\
\text { FDRs in } \\
\text { controls }\end{array}$ & $\begin{array}{l}\text { Oxford } 2011 \\
\text { level of } \\
\text { evidence }\end{array}$ & Observations \\
\hline \multicolumn{12}{|l|}{ AITD } \\
\hline & $\begin{array}{c}\text { Boelaert } 2010 \\
\text { [33] }\end{array}$ & UK & $\begin{array}{c}\text { Cross } \\
\text { sectional }\end{array}$ & 3286 & 845 & 6572 & & & & 3 & \\
\hline & $\begin{array}{l}\text { Hemminki } \\
2009 \text { [34] }\end{array}$ & Sweden & Cohort & 15,743 & 1,135 & 2,412 & & & & 3 & \\
\hline & $\begin{array}{c}\text { Criswell } 2005 \\
\text { [35] }\end{array}$ & USA & $\begin{array}{c}\text { Cross } \\
\text { sectional }\end{array}$ & 43 & 21 & 232 & & & & 3 & \\
\hline \multicolumn{12}{|l|}{ T1D } \\
\hline & $\begin{array}{c}\text { Criswell } 2005 \\
\text { [35] }\end{array}$ & USA & $\begin{array}{c}\text { Cross } \\
\text { sectional }\end{array}$ & 10 & 6 & 232 & & & & 3 & \\
\hline & $\begin{array}{c}\text { Bottazzo } 1978 \\
{[36]}\end{array}$ & UK & $\begin{array}{l}\text { Cross } \\
\text { sectional }\end{array}$ & 116 & 4 & 257 & & & & 3 & \\
\hline & $\begin{array}{c}\text { Anaya } 2006 \\
{[37]}\end{array}$ & Colombia & $C \& C$ & 98 & 18 & 312 & 113 & 9 & 362 & 3 & \\
\hline & $\begin{array}{l}\text { Wagner } 2011 \\
\text { [38] }\end{array}$ & Spain & $\begin{array}{c}\text { Cross } \\
\text { sectional }\end{array}$ & 12,973 & 97 & 1,279 & & & & 3 & Non diabetic siblings \\
\hline & & & & 12,973 & 1,001 & 6,262 & & & & & Diabetic siblings \\
\hline & $\begin{array}{l}\text { Hemminki } \\
2009 \text { [39] }\end{array}$ & Sweden & Cohort & 21,168 & 1,913 & 5,195 & & & & 3 & \\
\hline & $\begin{array}{l}\text { Samuelsson } \\
2004 \text { [40] }\end{array}$ & $\begin{array}{l}\text { Lithuania/ } \\
\text { Sweden }\end{array}$ & $C \& C$ & 803 & 114 & & 1,944 & 229 & & 3 & $N=$ for controls and for FDRs is not specified \\
\hline & $\begin{array}{l}\text { Lebenthal } \\
2011^{a}[41]\end{array}$ & Israel & $\begin{array}{c}\text { Cross } \\
\text { sectional }\end{array}$ & 121 & 57 & & & & & 3 & Familial T1D patients \\
\hline & & & & 226 & 43 & & & & & & Sporadic T1D patients. \\
\hline & & & & & & & & & & & Numbers of FDRs are not given \\
\hline \multicolumn{12}{|l|}{ SLE } \\
\hline & $\begin{array}{c}\text { Alarcón } \\
\text { Segovia } 2005 \\
{[42]}\end{array}$ & $\begin{array}{l}\text { Latin } \\
\text { america }\end{array}$ & $\begin{array}{l}\text { Cross } \\
\text { sectional }\end{array}$ & 1,177 & 50 & & & & & 3 & Total number of FDRs is not specified \\
\hline & $\begin{array}{l}\text { Priori } 2003 \\
\quad[43]\end{array}$ & Italy & $C \& C$ & 154 & 39 & 759 & 140 & 12 & 776 & 3 & \\
\hline & $\begin{array}{l}\text { Corporaal } \\
2002[44]\end{array}$ & $\begin{array}{l}\text { The } \\
\text { Netherlands }\end{array}$ & $\begin{array}{c}\text { Cross } \\
\text { sectional }\end{array}$ & 135 & 42 & 693 & & & & 3 & \\
\hline & $\begin{array}{c}\text { Criswell } 2005 \\
\text { [35] }\end{array}$ & USA & $\begin{array}{l}\text { Cross } \\
\text { sectional }\end{array}$ & 65 & 47 & 232 & & & & 3 & \\
\hline \multicolumn{12}{|l|}{ RA } \\
\hline & $\begin{array}{c}\text { Criswell } 2005 \\
\text { [35] }\end{array}$ & USA & $\begin{array}{c}\text { Cross } \\
\text { sectional }\end{array}$ & 46 & 31 & 232 & & & & 3 & \\
\hline & Lin 1998 [45] & USA & $C \& C$ & 29 & 25 & 218 & 14 & 4 & 98 & 3 & \\
\hline & $\begin{array}{c}\text { Thomas } 1983 \\
{[46]}\end{array}$ & UK & $C \& C$ & 295 & 19 & 2,081 & 307 & 8 & 2,299 & 3 & \\
\hline
\end{tabular}


Table 2 Characteristics of the studies included. (Continued)

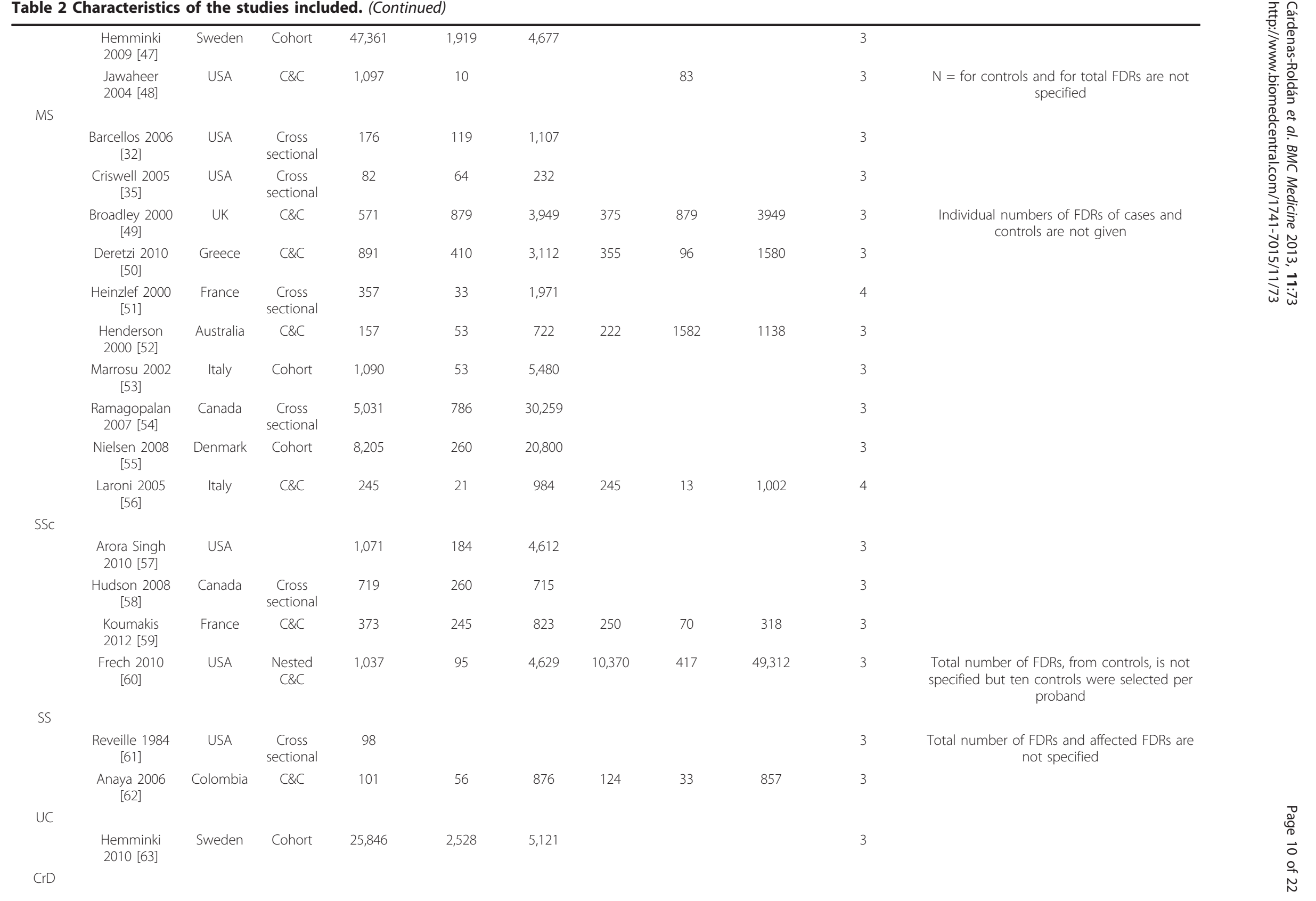


Table 2 Characteristics of the studies included. (Continued)

\begin{tabular}{|c|c|c|c|c|c|c|c|c|c|c|c|}
\hline & $\begin{array}{l}\text { Hemminki } \\
2010[63]\end{array}$ & Sweden & Cohort & 18,885 & 2,169 & 4,306 & & & & 3 & \\
\hline \multicolumn{12}{|l|}{ IBD } \\
\hline & $\begin{array}{c}\text { Criswell } 2005 \\
\text { [35] }\end{array}$ & USA & $\begin{array}{c}\text { Cross } \\
\text { sectional }\end{array}$ & 7 & 8 & 232 & & & & 3 & \\
\hline \multicolumn{12}{|l|}{ VIT } \\
\hline & $\begin{array}{l}\text { Alkhateeb } \\
2003 \text { [64] }\end{array}$ & USA/UK & $\begin{array}{l}\text { Cross } \\
\text { sectional }\end{array}$ & 2,624 & 660 & 8,034 & & & & 3 & \\
\hline & $\begin{array}{l}\text { Laberge } 2005 \\
\quad[65]\end{array}$ & USA & $\begin{array}{l}\text { Cross } \\
\text { sectional }\end{array}$ & 133 & 98 & 331 & & & & 3 & \\
\hline & $\begin{array}{l}\text { Zhang } 2009 \\
{[66]}\end{array}$ & China & $\begin{array}{c}\text { Cross } \\
\text { sectional }\end{array}$ & 5,601 & 340 & 18,705 & & & & 3 & \\
\hline \multicolumn{12}{|l|}{ JRA } \\
\hline & $\begin{array}{c}\text { Prahalad } 2002 \\
\text { [67] }\end{array}$ & USA & $C \& C$ & 110 & 72 & 446 & 45 & 29 & 181 & 3 & \\
\hline & $\begin{array}{l}\text { Huang } 2004 \\
{[68]}\end{array}$ & Taiwan & $\begin{array}{l}\text { Cross } \\
\text { sectional }\end{array}$ & 110 & 5 & & & & & 3 & Total number of FDRs is not specified \\
\hline \multicolumn{12}{|l|}{ JSLE } \\
\hline & $\begin{array}{l}\text { Huang } 2004 \\
\quad[68]\end{array}$ & Taiwan & $\begin{array}{l}\text { Cross } \\
\text { sectional }\end{array}$ & 91 & 6 & & & & & 3 & Total number of FDRs is not specified \\
\hline & $\begin{array}{l}\text { Walters } 2012 \\
\quad[69]\end{array}$ & USA & $\begin{array}{l}\text { Cross } \\
\text { sectional }\end{array}$ & 69 & 23 & & & & & 3 & Total number of FDRs is not specified \\
\hline \multicolumn{12}{|l|}{ IIM } \\
\hline & $\begin{array}{l}\text { Ginn } 1998 \\
\quad[70]\end{array}$ & USA & $C \& C$ & 21 & 33 & 151 & 21 & 7 & 143 & 3 & \\
\hline & $\begin{array}{c}\text { Niewold } 2011 \\
{[71]}\end{array}$ & USA & $\begin{array}{l}\text { Cross } \\
\text { sectional }\end{array}$ & 304 & 30 & 1,224 & & & & 3 & \\
\hline \multicolumn{12}{|l|}{$C D$} \\
\hline & $\begin{array}{l}\text { Petaros } 2002 \\
\quad[72]\end{array}$ & Italy & $C \& C$ & 125 & 18 & 373 & 125 & 4 & 352 & 3 & \\
\hline & $\begin{array}{l}\text { Cataldo } 2003 \\
\quad[73]\end{array}$ & Italy & $C \& C$ & 66 & 11 & 225 & 68 & 2 & 232 & 3 & \\
\hline & $\begin{array}{l}\text { Neuhausen } \\
2008[74]\end{array}$ & USA & $\begin{array}{c}\text { Cross } \\
\text { sectional }\end{array}$ & 408 & 58 & 1,272 & & & & 3 & \\
\hline \multicolumn{12}{|l|}{$\mathrm{AA}$} \\
\hline & $\begin{array}{l}\text { Kakouroru } \\
2007[75]\end{array}$ & Greece & $C \& C$ & 157 & & & 100 & & & 3 & $\begin{array}{c}\text { Total number of FDRs and affected FDRs are } \\
\text { not specified }\end{array}$ \\
\hline \multicolumn{12}{|r|}{ 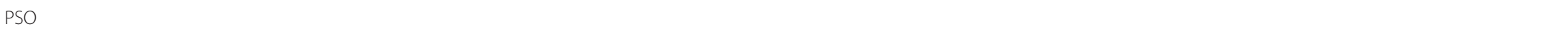 } \\
\hline & $\begin{array}{l}\text { Criswell } 2005 \\
{[35]}\end{array}$ & USA & $\begin{array}{c}\text { Cross } \\
\text { sectional }\end{array}$ & 8 & 8 & 232 & & & & 3 & \\
\hline
\end{tabular}

${ }^{a}$ Numbers depicted represent families and not family members. Note: Only classical ADs are taken into account. Although some studies did not report the number of FDRs they specified other measures of

association. See text for more details. AA, alopecia areata; AITD, autoimmune thyroid disease; C\&C, case control; CD, celiac disease; CrD, Crohn's disease; IBD, inflammatory bowel disease; IIM, idiopathic inflammatory myositis; JRA, juvenile rheumatoid arthritis; JSLE, juvenile systemic lupus erythematosus; MS, multiple sclerosis; PSO, psoriasis; RA, rheumatoid arthritis; SLE, systemic lupus erythematosus; SS, Sjögren's syndrome; SSC, systemic sclerosis; T1D, type 1 diabetes; UC, ulcerative colitis; VIT, vitiligo. 


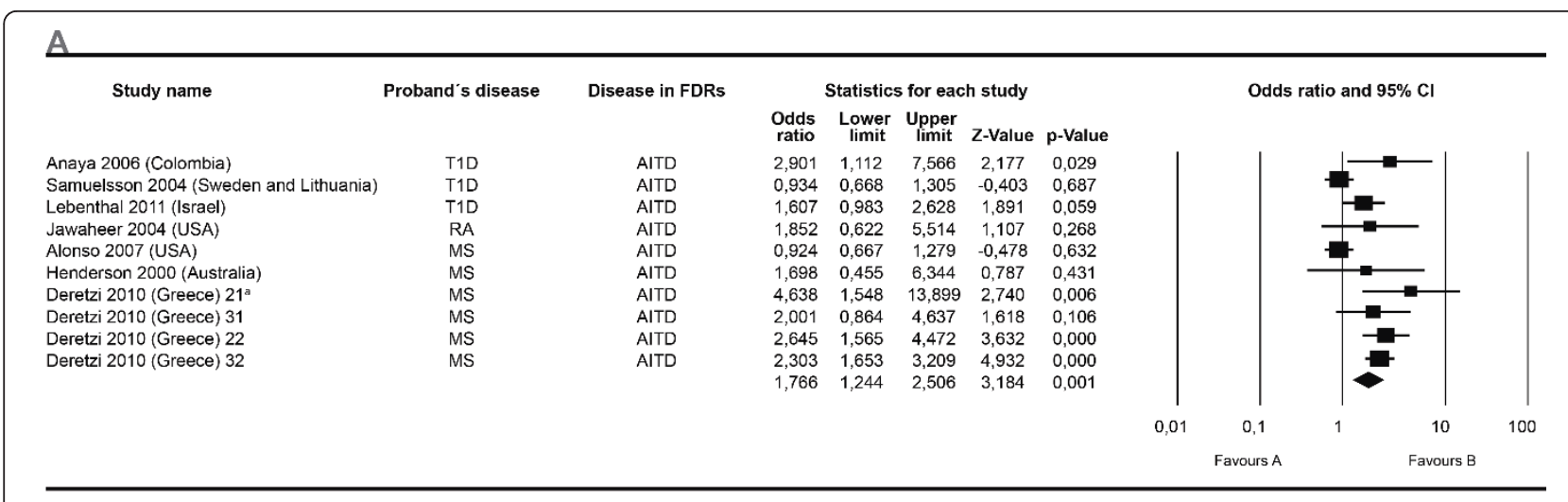

B

\begin{tabular}{|c|c|c|c|c|c|c|c|}
\hline Alonso 2007 (USA) & MS & T1D & 0,797 & 0,577 & 1,100 & $-1,381$ & 0,167 \\
\hline Deretzi 2010 (Greece) 29a & MS & T1D & 7,215 & 2,485 & 20,946 & 3,634 & 0,000 \\
\hline Deretzi 2010 (Greece) 39 & MS & T1D & 2,708 & 1,122 & 6,537 & 2,216 & 0,027 \\
\hline Henderson 2000 (Australia) & MS & T1D & 1,415 & 0,361 & 5,545 & 0,498 & 0,618 \\
\hline Marrosu 2002 (Italy) & MS & T1D & 4,113 & 2,164 & 7,818 & 4,315 & 0,000 \\
\hline Marrosu 2002 (Italy) & MS & T1D & 1,234 & 0,769 & 1,980 & 0,871 & 0,384 \\
\hline Ramagopalan 2007 (Canada) & MS & T1D & 0,709 & 0,406 & 1,238 & $-1,208$ & 0,227 \\
\hline \multirow[t]{2}{*}{ Thomas 1983 (UK) } & RA & T1D & 2,624 & 1,146 & 6,006 & 2,283 & 0,022 \\
\hline & & & 1,822 & 1,047 & 3,171 & 2,124 & 0,03 \\
\hline
\end{tabular}

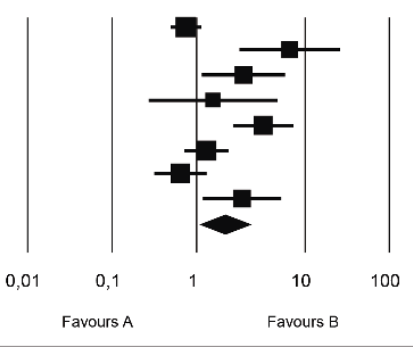

C

\begin{tabular}{llllllll}
\hline & & & & & & \\
Deretzi 2010 (Greece) 23a & MS & IBD & 4,810 & 1,841 & 12,568 & 3,205 & 0,001 \\
Deretzi 2010 (Greece) 33 & MS & IBD & 3,237 & 1,591 & 6,586 & 3,242 & 0,001 \\
Henderson 2000 (Australia) & MS & IBD & 0,566 & 0,100 & 3,216 & $-0,642$ & 0,521 \\
Ramagopalan 2007 (Canada) & MS & IBD & 1,264 & 0,458 & 3,485 & 0,452 & 0,651 \\
Ramagopalan 2007 (Canada) & MS & IBD & 1,951 & 0,716 & 5,317 & 1,306 & 0,192 \\
& & & 2,227 & 1,223 & 4,056 & 2,617 & 0,009
\end{tabular}

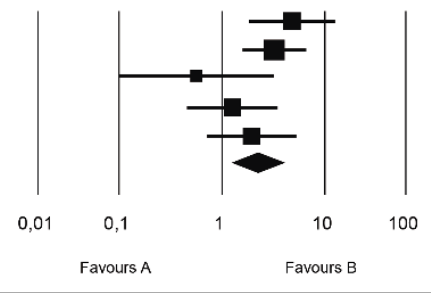

D

Study name

Henderson 2000 (Australia)

Magaña 2012 (Mexico)

Priori 2003 (Italy)

\begin{tabular}{cl} 
Proband's disease & \multicolumn{1}{c}{ Outcome } \\
MS & Familial autoimmunity \\
MS & Familial autoimmunity \\
SLE & Familial autoimmunity
\end{tabular}

Statistics for each study

Odds Lower Upper

ratio limit limit Z-Value $p$-Value

$\begin{array}{lllll}2,200 & 1,304 & 3,712 & 2,955 & 0,003\end{array}$

$\begin{array}{llllll}2,125 & 0,865 & 5,223 & 1,643 & 0,100\end{array}$

$\begin{array}{llllll}3,977 & 1,785 & 8,864 & 3,376 & 0,001\end{array}$

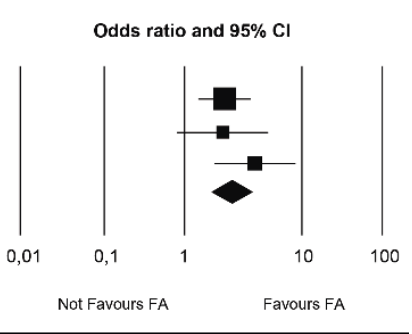

Not Favours FA

Figure 4 Forest plots depicting odds ratios for specific autoimmune diseases in first degree relatives. Familial autoimmunity has to be seen as a two way relationship depending on which member of the nuclear family is the proband. Therefore, grouping meta-analysis by the disease present in FDRs is equivalent to analyzing it by the disease present in the proband. The figure shows four different analyses. From top to bottom autoimmune thyroid disease (A), type 1 diabetes mellitus (B), inflammatory bowel disease (C) and familial autoimmunity (D) assessed as an outcome. The summary effect (random effect model) is depicted as a diamond at the bottom of each analysis. The lateral points of each diamond indicate confidence intervals for this estimate. ${ }^{a}$ Numbers represent different subgroups within the study.

corresponding funnel plot showing the standard error or the precision on the $\mathrm{Y}$ axis is shown in Additional file 2. Therefore, a second analysis was run in a search for publication bias. The classic fail-safe analysis indicated a number of missing studies that would give a $P$-value of $>0.05$. Begg and Mazumdar rank correlation was not significant and the trim and fill adjustment did not suggest a lower risk than the original analysis. Based on all 


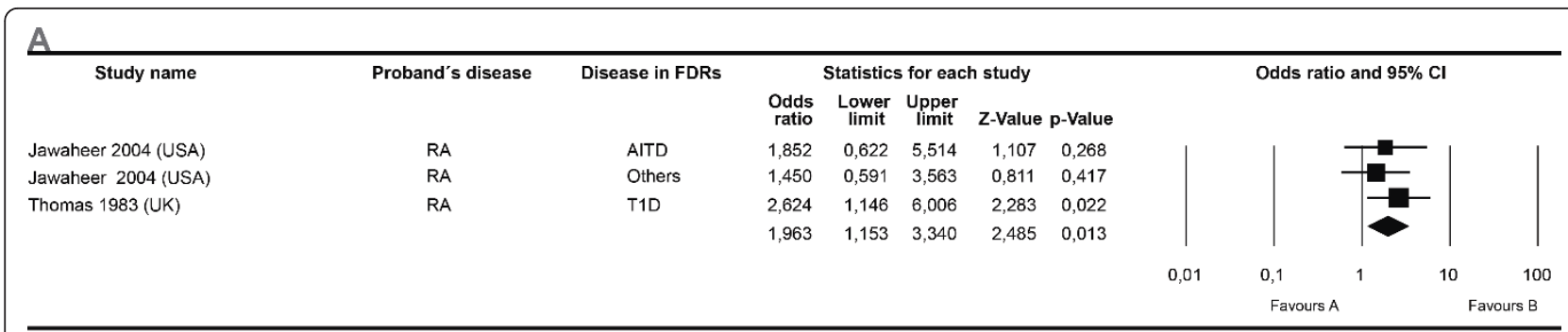

\section{B}

Alonso 2007 (USA)

Alonso 2007 (USA)

Alonso 2007 (USA)

Alonso 2007 (USA)

Alonso 2007 (USA)

Deretzi 2010 (Greece) 21 ${ }^{a}$

Deretzi 2010 (Greece) 22

Deretzi 2010 (Greece) 23

Deretzi 2010 (Greece) 24

Deretzi 2010 (Greece) 25

Deretzi 2010 (Greece) 26

Deretzi 2010 (Greece) 27

Deretzi 2010 (Greece) 28

Deretzi 2010 (Greece) 29

Deretzi 2010 (Greece) 30

Deretzi 2010 (Greece) 31

Deretzi 2010 (Greece) 32

Deretzi 2010 (Greece) 33

Deretzi 2010 (Greece) 34

Deretzi 2010 (Greece) 35

Deretzi 2010 (Greece) 36

Deretzi 2010 (Greece) 37

Deretzi 2010 (Greece) 38

Deretzi 2010 (Greece) 39

Deretzi 2010 (Greece) 40

Henderson 2000 (Australia)

Henderson 2000 (Australia)

Henderson 2000 (Australia)

Henderson 2000 (Australia)

Henderson 2000 (Australia)

Henderson 2000 (Australia)

Henderson 2000 (Australia)

Henderson 2000 (Australia)

Henderson 2000 (Australia)

Henderson 2000 (Australia)

Henderson 2000 (Australia)

Marrosu 2002 (Italy)

Marrosu 2002 (Italy)

Ramagopalan 2007 (Canada)

Ramagopalan 2007 (Canada)

Ramagopalan 2007 (Canada)

Ramagopalan 2007 (Canada)

\begin{tabular}{|c|c|}
\hline MS & Any AD (no MS) \\
\hline MS & AITD \\
\hline MS & RA \\
\hline MS & SLE \\
\hline MS & T1D \\
\hline MS & AITD \\
\hline MS & AITD \\
\hline MS & IBD \\
\hline MS & MG \\
\hline MS & PSO \\
\hline MS & RA \\
\hline MS & SLE \\
\hline MS & SS \\
\hline MS & T1D \\
\hline MS & VIT \\
\hline MS & AITD \\
\hline MS & AITD \\
\hline MS & IBD \\
\hline MS & MG \\
\hline MS & PSO \\
\hline MS & RA \\
\hline MS & SLE \\
\hline MS & ss \\
\hline MS & T1D \\
\hline MS & VIT \\
\hline MS & AS \\
\hline MS & AITD \\
\hline MS & AITD \\
\hline MS & IBD \\
\hline MS & PBC \\
\hline MS & PSO \\
\hline MS & RA \\
\hline MS & SLE \\
\hline MS & SSc \\
\hline MS & T1D \\
\hline MS & VIT \\
\hline MS & T1D /MS multiplex \\
\hline MS & T1D /MS simplex \\
\hline MS & $\mathrm{CrD}$ \\
\hline MS & RA \\
\hline MS & T1D \\
\hline
\end{tabular}

$\begin{array}{llllll}0,909 & 0,721 & 1,145 & -0,813 & 0,416\end{array}$

$\begin{array}{llllll}0,924 & 0,667 & 1,279 & -0,478 & 0,632\end{array}$

$\begin{array}{lllll}0,961 & 0,698 & 1,322 & -0,246 & 0,806\end{array}$

$\begin{array}{lllll}1,476 & 0,824 & 2,645 & 1,310 & 0,190\end{array}$

$\begin{array}{lllll}0,797 & 0,577 & 1,100 & -1,381 & 0,167\end{array}$

$\begin{array}{llllll}4,638 & 1,548 & 13,899 & 2,740 & 0,006\end{array}$

$\begin{array}{lllll}2,645 & 1,565 & 4,472 & 3,632 & 0,000\end{array}$

$\begin{array}{lllll}4,810 & 1,841 & 12,568 & 3,205 & 0,001\end{array}$

$\begin{array}{llllll}5,411 & 0,337 & 86,751 & 1,193 & 0,233\end{array}$

$\begin{array}{llllll}4,810 & 1,841 & 12,568 & 3,205 & 0,001\end{array}$

$\begin{array}{lllll}2,705 & 0,493 & 14,839 & 1,146 & 0,252\end{array}$

$\begin{array}{llllll}1,804 & 0,485 & 6,702 & 0,881 & 0,378\end{array}$

$\begin{array}{lllll}3,607 & 0,600 & 21,683 & 1,402 & 0,161\end{array}$

$\begin{array}{llllll}7,215 & 2,485 & 20,946 & 3,634 & 0,000\end{array}$

$\begin{array}{llllll}5,411 & 0,337 & 86,751 & 1,193 & 0,233\end{array}$

$\begin{array}{llllll}2,001 & 0,864 & 4,637 & 1,618 & 0,106\end{array}$

$\begin{array}{lllll}2,303 & 1,653 & 3,209 & 4,932 & 0,000\end{array}$

$\begin{array}{lllll}3,237 & 1,591 & 6,586 & 3,242 & 0,001\end{array}$

$\begin{array}{llllll}0,560 & 0,035 & 8,964 & -0,410 & 0,682\end{array}$

$\begin{array}{llllll}1,556 & 0,725 & 3,343 & 1,134 & 0,257\end{array}$

$\begin{array}{llllll}0,700 & 0,188 & 2,612 & -0,530 & 0,596\end{array}$

$\begin{array}{llllll}1,058 & 0,471 & 2,380 & 0,137 & 0,891\end{array}$

$\begin{array}{llllll}0,934 & 0,223 & 3,913 & -0,094 & 0,925\end{array}$

$\begin{array}{lllll}2,708 & 1,122 & 6,537 & 2,216 & 0,027\end{array}$

$\begin{array}{llllll}0,560 & 0,035 & 8,964 & -0,410 & 0,682\end{array}$

$\begin{array}{llllll}6,792 & 0,792 & 58,254 & 1,747 & 0,081\end{array}$

$\begin{array}{lllll}1,698 & 0,455 & 6,344 & 0,787 & 0,431\end{array}$

$\begin{array}{llllll}0,970 & 0,307 & 3,069 & -0,051 & 0,959\end{array}$

$\begin{array}{llllll}0,566 & 0,100 & 3,216 & -0,642 & 0,521\end{array}$

$\begin{array}{llllll}0,377 & 0,015 & 9,450 & -0,594 & 0,553\end{array}$

$\begin{array}{llllll}0,599 & 0,247 & 1,457 & -1,129 & 0,259\end{array}$

$\begin{array}{llllll}0,889 & 0,372 & 2,127 & -0,263 & 0,792\end{array}$

$\begin{array}{llllll}0,970 & 0,307 & 3,069 & -0,051 & 0,959\end{array}$

$\begin{array}{llllll}3,393 & 0,135 & 85,051 & 0,743 & 0,457\end{array}$

$\begin{array}{lllll}1,415 & 0,361 & 5,545 & 0,498 & 0,618\end{array}$

$\begin{array}{lllll}1,132 & 0,069 & 18,548 & 0,087 & 0,931\end{array}$

$\begin{array}{lllll}4,113 & 2,164 & 7,818 & 4,315 & 0,000\end{array}$

$\begin{array}{llllll}1,234 & 0,769 & 1,980 & 0,871 & 0,384\end{array}$

$\begin{array}{lllll}1,264 & 0,458 & 3,485 & 0,452 & 0,651\end{array}$

$\begin{array}{llllll}0,711 & 0,549 & 0,921 & -2,586 & 0,010\end{array}$

$\begin{array}{llllll}0,709 & 0,406 & 1,238 & -1,208 & 0,227\end{array}$

$\begin{array}{llllll}1,951 & 0,716 & 5,317 & 1,306 & 0,192\end{array}$

$\begin{array}{lllll}1,522 & 1,230 & 1,883 & 3,863 & 0,000\end{array}$

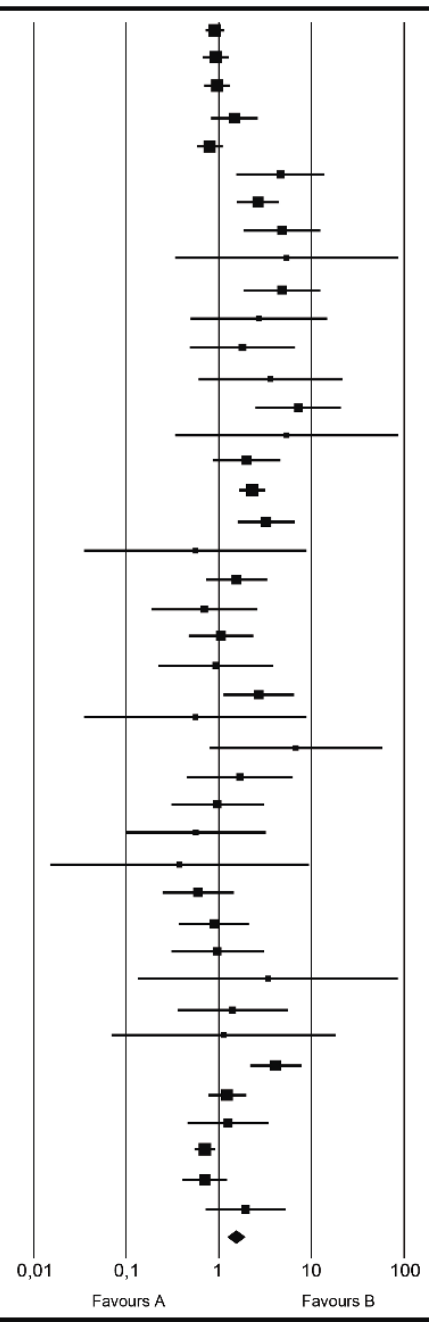

Figure 5 Forest plots depicting odds ratios for familial autoimmunity. The figure shows two different analyses. From top to bottom: (A) rheumatoid arthritis (RA), (B) multiple sclerosis (MS). Autoimmune diseases in first degree relatives through all the studies from a specific autoimmune disease present in the proband were analyzed. The summary effect (random effect model) is depicted as a diamond at the bottom

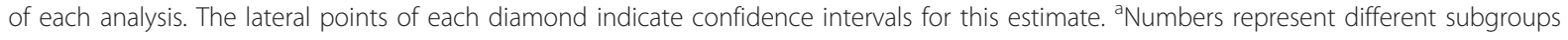
within the study.

the analyses for publication bias, we consider the impact of bias in the three meta-analyses to be trivial.

Familial autoimmunity as an outcome was also assessed in certain articles, particularly in MS and SLE probands (Figure 4D).
Aggregation

Several studies retrieved only reported prevalences of ADs in FDRs. Aggregation, based on data from five studies mentioned earlier in Table 3[2,29-32], is shown in Table 4, which discloses information on calculated 


\begin{tabular}{|c|c|c|c|c|c|c|c|}
\hline \multirow[t]{2}{*}{ Study name } & \multirow[t]{2}{*}{ Proband's disease } & \multirow[t]{2}{*}{ Disease in FDRs } & \multicolumn{5}{|c|}{ Statistics for each study } \\
\hline & & & $\begin{array}{c}\text { Risk } \\
\text { ratio }\end{array}$ & $\begin{array}{c}\text { Lower } \\
\text { limit }\end{array}$ & $\begin{array}{c}\text { Upper } \\
\text { limit }\end{array}$ & z-Value & ep-Value \\
\hline Boelaert $2010(\mathrm{UK}) 1^{3}$ & AITD & $\mathrm{AdD}$ & 5,970 & 1,585 & 22,480 & 2,641 & 0,008 \\
\hline Boelaert 2010 (UK) 14 & AITD & $C D$ & 5,730 & 3,403 & 9,648 & 6,566 & \\
\hline Boelaert 2010 (UK) 17 & AITD & IBD & 2,340 & 1,647 & 3,325 & 4,745 & \\
\hline $\begin{array}{l}\text { Boelaert 2010 (UKK) } 18 \\
\text { Boeleart } 2010 \text { (UK) } 20\end{array}$ & AITD & $M G$ & $\begin{array}{r}2,390 \\
14,100\end{array}$ & 0,438 & 13,038 & 1,007 & \\
\hline $\begin{array}{l}\text { Boelearet 2010 (UKK) } 20 \\
\text { Boelaert } 2010 \text { (UK) } 21\end{array}$ & & ${ }_{B A}^{P A}$ & & & & & \\
\hline $\begin{array}{l}\text { Beelaert } 2010 \text { (UK) } 21 \\
\text { Boelaert } 2010 \text { (UK) } 22\end{array}$ & $\begin{array}{l}\text { ATTD } \\
\text { AITD }\end{array}$ & $\begin{array}{l}\text { RA } \\
S L E\end{array}$ & $\begin{array}{l}13,550 \\
4650\end{array}$ & $\begin{array}{l}12,338 \\
2057\end{array}$ & $\begin{array}{l}14,881 \\
10514\end{array}$ & $\begin{array}{l}54,505 \\
3692\end{array}$ & 50,000 \\
\hline Boelaert 2010 (UK) 23 & AITD & T1D & 3,640 & 2,858 & $\begin{array}{c}10,514 \\
4,636\end{array}$ & $\begin{array}{l}3,042 \\
10472\end{array}$ & 20,000 \\
\hline Boelaert 2010 (UK) 24 & AITD & VIT & 6,090 & 4,291 & 8,643 & 10,114 & \\
\hline Boelaert 2010 (UK) 38 & AITD & $C D$ & 6,100 & 1,623 & 22,928 & 2,677 & 0,007 \\
\hline Boelaert 2010 (UK) 41 & AITD & IBD & 1,170 & 0,311 & 4,404 & 0,232 & 0,816 \\
\hline $\begin{array}{l}\text { Boelaert 2010 (UK) } 44 \\
\text { Boeleart } 2010 \text { (UK) } 45\end{array}$ & $\begin{array}{l}\text { AITD } \\
\text { ATTD }\end{array}$ & ${ }_{\mathrm{RA}}^{\mathrm{PA}}$ & $\begin{array}{l}{ }_{15,540}^{13,040} \\
-1\end{array}$ & $\begin{array}{r}9,819 \\
10,349\end{array}$ & $\begin{array}{l}24,594 \\
16,431\end{array}$ & $\begin{array}{r}11,712 \\
21775\end{array}$ & 0,000 \\
\hline $\begin{array}{l}\text { Boelaert 2010 (UK) } 45 \\
\text { Boeleart } 2010 \text { (UK) } 46\end{array}$ & $\begin{array}{l}\text { ATTD } \\
\text { AITD }\end{array}$ & $\begin{array}{l}\text { RA } \\
\text { SLE }\end{array}$ & $\begin{array}{l}13,040 \\
7,480\end{array}$ & $\begin{array}{l}10,349 \\
2,022\end{array}$ & $\begin{array}{l}16,431 \\
27,671\end{array}$ & $\begin{array}{l}21,775 \\
3,015\end{array}$ & $\begin{array}{l}0,000 \\
0,003\end{array}$ \\
\hline Boelaert 2010 (UK) 47 & $\begin{array}{l}\text { AlID } \\
\text { AITD }\end{array}$ & T1D & $\begin{array}{r}7,480 \\
7,430\end{array}$ & $\begin{array}{l}2,022 \\
4,946\end{array}$ & $\begin{array}{l}27,6 / 1 \\
11,162\end{array}$ & $\begin{array}{l}3,15 \\
9,659\end{array}$ & 0,000 \\
\hline Boelaert 2010 (UK) 48 & AITD & VIT & 8,080 & 3,796 & 17,200 & 5,420 & 0,000 \\
\hline Hemminki 2009 (Sweden) AITD10 & AITD & $\mathrm{AdD}$ & 3,060 & 1,055 & 8,873 & 2,059 & 0,039 \\
\hline Hemminki 2009 (Sweden) AITD12 & AITD & AS & 1,160 & 0,677 & 1,988 & 0,540 & 0,589 \\
\hline Hemminki 2009 (Sweden) AITD13 & AITD & AS & 0,990 & 0,390 & 2,516 & $-0,021$ & 0,983 \\
\hline $\begin{array}{l}\text { Hemminkink } 2009 \text { (Sweden) A AlT14 } \\
\text { Hemminki } 2009 \text { Sweden) AlTD 15 }\end{array}$ & AITD & HA & 1,760 & 0,647 & $\begin{array}{l}4,785 \\
2334\end{array}$ & 1,108 & 0,268 \\
\hline $\begin{array}{l}\text { Hemminkik } 2009 \text { (Sweden) A AlTD 15 } \\
\text { Hemminki } 2009 \text { (Sweden) AlTD16 }\end{array}$ & $\begin{array}{l}\text { ATTD } \\
\text { ATTD }\end{array}$ & $\begin{array}{l}C D \\
C D\end{array}$ & $\begin{array}{l}1,1,00 \\
1,300\end{array}$ & $\begin{array}{l}0,512 \\
0,546\end{array}$ & $\begin{array}{l}2,364 \\
3,093\end{array}$ & $\begin{array}{l}0,244 \\
0,593\end{array}$ & $\begin{array}{l}0,807 \\
0,553\end{array}$ \\
\hline Hemminki 2009 (Sweden) AITD 18 & $\begin{array}{l}\text { AllD } \\
\text { AITD }\end{array}$ & $\mathrm{CrD}$ & $\begin{array}{l}1,3000 \\
0,950\end{array}$ & $\begin{array}{l}0,546 \\
0,662\end{array}$ & $\begin{array}{l}3,393 \\
1,364\end{array}$ & $\begin{array}{r}-0,278 \\
-0,29\end{array}$ & 0,781 \\
\hline Hemminki 2009 (Sweden) AlTD 19 & AITD & $\mathrm{CrD}$ & 1,140 & 0,613 & 2,120 & 0,414 & 0,679 \\
\hline Hemminki 2009 (Sweden) AITD20 & AITD & T1D & 3,370 & 1,233 & 9,212 & 2,368 & 0,018 \\
\hline Hemminki 2009 (Sweden) AITD23 & AITD & DISCOID LUPUS & 2,620 & 0,831 & 8,261 & 1,644 & 0,100 \\
\hline Hemminki 2009 (Sweden) AIT & AITD & ITP & 1,180 & 0,518 & $\begin{array}{l}2,689 \\
5,009\end{array}$ & 0,394 & 0,694 \\
\hline $\begin{array}{l}\text { Hemmininki } 2009 \text { (Sweden) AIT A } \\
\text { Hemminki } 200 \text { (Swede) AIT }\end{array}$ & $\begin{array}{l}\text { ATTD } \\
\text { ATTD }\end{array}$ & ITP & $\begin{array}{l}1,830 \\
1190\end{array}$ & 0,633 & $\begin{array}{l}5,290 \\
470\end{array}$ & 1,116 & $\begin{array}{l}0,264 \\
0,806\end{array}$ \\
\hline Hemmininki 2009 (Sweden) AITL & $\begin{array}{l}\text { ATTD } \\
\text { AITD }\end{array}$ & $\begin{array}{l}\text { SSC } \\
\text { DISCOID LUPUS }\end{array}$ & $\begin{array}{l}1,1,00 \\
6,030\end{array}$ & $\begin{array}{l}0,298 \\
1,564\end{array}$ & $\begin{array}{l}4,760 \\
23,250\end{array}$ & $\begin{array}{l}0,246 \\
2609\end{array}$ & $\begin{array}{l}0,806 \\
0,009\end{array}$ \\
\hline Hemminki 2009 (Sweden) AITL & AITD & MG & 2,040 & $\begin{array}{l}1,004 \\
1,160\end{array}$ & 3,586 & 2,477 & 0,013 \\
\hline Hemminki 2009 (Sweden) AITD33 & AITD & MG & 1,590 & 0,453 & 5,582 & 0,724 & 0,469 \\
\hline Hemminki 2009 (Sweden) AITD35 & AITD & PAN & 1,600 & 0,651 & 3,930 & 1,025 & 0,305 \\
\hline Hemminki 2009 (Sweden) AITC & AITD & $\lim$ & $\begin{array}{l}2,480 \\
2,80\end{array}$ & 1,207 & $\begin{array}{l}5,097 \\
0,981\end{array}$ & 2,471 & 0,013 \\
\hline Hemminki 2009 (Sweden) Al] & AITD & $\operatorname{IIM}$ & 2,820 & 0,809 & 9,831 & 1,627 & 0,104 \\
\hline $\begin{array}{l}\text { Hemminki } 2009 \text { (Sweden AI } \\
\text { Hemminki } 2009 \text { Swweden Al }\end{array}$ & $\begin{array}{l}\text { AlTD } \\
\text { ATTD }\end{array}$ & $\begin{array}{l}P B C \\
\text { SSC }\end{array}$ & 0,930 & 0,294 & $\begin{array}{r}2,941 \\
25535\end{array}$ & $-0,124$ & $\begin{array}{l}0,902 \\
0,006\end{array}$ \\
\hline 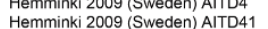 & $\begin{array}{l}\text { AlID } \\
\text { AITD }\end{array}$ & $\begin{array}{l}\text { SSc } \\
\text { PSO }\end{array}$ & $\begin{array}{l}6,620 \\
11090\end{array}$ & $\begin{array}{l}1,716 \\
0818\end{array}$ & $\begin{array}{l}25,535 \\
1.53\end{array}$ & $\begin{array}{l}2,444 \\
0,587\end{array}$ & $\begin{array}{l}0,006 \\
0,557\end{array}$ \\
\hline Hemminki 2009 (Sweden) AITD42 & AITD & PSO & 1,070 & 0,525 & $\begin{array}{l}1,143 \\
2,181\end{array}$ & 0,186 & 0,852 \\
\hline Hemminki 2009 (Sweden) AITD44 & AITD & $\mathrm{RA}$ & 1,380 & 0,765 & 2,488 & 1,071 & 0.284 \\
\hline Hemminki 2009 (Sweden) AITD47 & AITD & ss & 1,230 & 0,451 & 3,354 & 0,404 & 0,686 \\
\hline i) 2009 (Sweden) AIT! & AITD & ss & 1,010 & 0,117 & 8,739 & 0,009 & 0,993 \\
\hline Hemminki 2009 (Sweden) AITC & AITD & SLE & 1,840 & 0,770 & 4,399 & 1,371 & 0,170 \\
\hline $\begin{array}{l}\text { Hemmink } \\
\text { Hemmink }\end{array}$ & $\begin{array}{l}\text { AlTD } \\
\text { ATTD }\end{array}$ & $T_{S 1 D}^{T D}$ & 2,140 & $\begin{array}{l}1,172 \\
0,865\end{array}$ & $\begin{array}{l}3,909 \\
2,266\end{array}$ & $\begin{array}{l}2,475 \\
1,37\end{array}$ & $\begin{array}{l}0,013 \\
0,171\end{array}$ \\
\hline $\begin{array}{l}\text { Hemminkk } 2009 \text { (Sweden) A AlIIS0 } \\
\text { Hemminki } 2009 \text { (Swen) AITD }\end{array}$ & $\begin{array}{l}\text { AllD } \\
\text { AITD }\end{array}$ & $\begin{array}{l}\text { SLE } \\
\text { SSC }\end{array}$ & $\begin{array}{l}1,4,300 \\
1,340\end{array}$ & $\begin{array}{l}0,865 \\
0.818\end{array}$ & $\begin{array}{l}2,266 \\
2,195\end{array}$ & $\begin{array}{l}1,310 \\
1,162\end{array}$ & $\begin{array}{l}0,171 \\
0,245\end{array}$ \\
\hline inki 2009 (Sweden) AITL & AITD & SSC & $\begin{array}{r}1,340 \\
1,370\end{array}$ & 0,492 & 3,817 & 0,602 & $\begin{array}{l}0,245 \\
0,547\end{array}-2$ \\
\hline inki 2009 (Sweden) AITL & AITD & UC & 1,300 & 1,017 & 1,662 & 2,095 & 0,036 \\
\hline i 2009 (Sweden) Al & AITD & UC & 1,390 & 0,794 & 2,433 & 1,153 & \\
\hline (Swe & AITD & WG & 1,250 & 0,926 & 1,688 & 1,455 & \\
\hline 9 (Swe & AIT & WG & 1,310 & 0,293 & 5,852 & 0,354 & \\
\hline 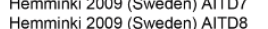 & $\begin{array}{l}\text { AATD } \\
\text { AITD }\end{array}$ & $\begin{array}{l}\text { PA } \\
\text { RA }\end{array}$ & $\begin{array}{l}1,820 \\
1,480\end{array}$ & $\begin{array}{l}1,333 \\
1,311\end{array}$ & $\begin{array}{l}2,485 \\
1,71\end{array}$ & $\begin{array}{l}3,771 \\
6329\end{array}$ & \\
\hline Hemminki 2009 (Sweden) AITD9 & AITD & $\mathrm{AdD}$ & $\begin{array}{l}2,400 \\
2,520\end{array}$ & 1,227 & 5,176 & 2,517 & \\
\hline & & & 2,421 & 1,749 & 3,351 & 5,331 & 0,000 \\
\hline
\end{tabular}

Risk ratio and $95 \% \mathrm{Cl}$

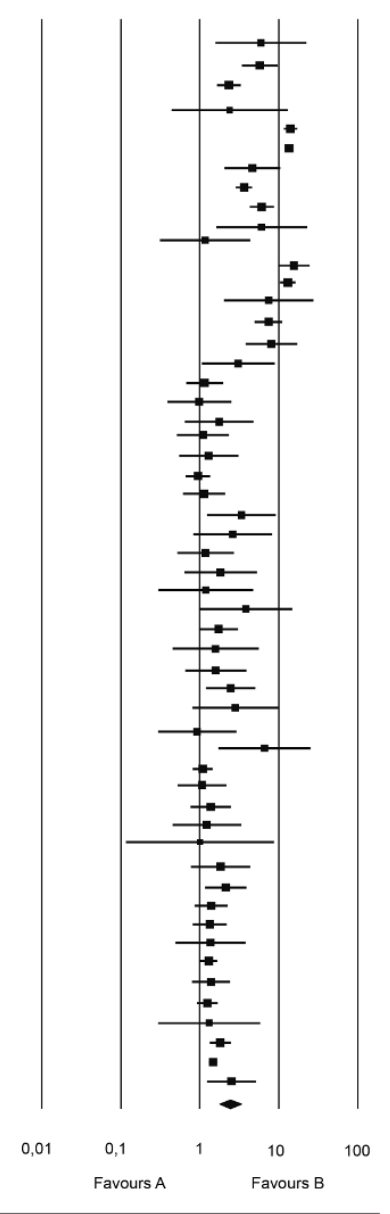

B

$\begin{array}{ll}\text { MS } & \text { AdD } \\ \text { MS } & \text { AS } \\ \text { MS } & \text { CD } \\ \text { MS } & \text { CrD } \\ \text { MS } & \text { HA } \\ \text { MS } & \text { AITD } \\ \text { MS } & \text { JIA } \\ \text { MS } & \text { MG } \\ \text { MS } & \text { PA } \\ \text { MS } & \text { PAN } \\ \text { MS } & \text { PBC } \\ \text { MS } & \text { PSO } \\ \text { MS } & \text { RA } \\ \text { MS } & \text { SLE } \\ \text { MS } & \text { SS } \\ \text { MS } & \text { SSC } \\ \text { MS } & \text { MC } \\ \text { MS } & \text { WG }\end{array}$

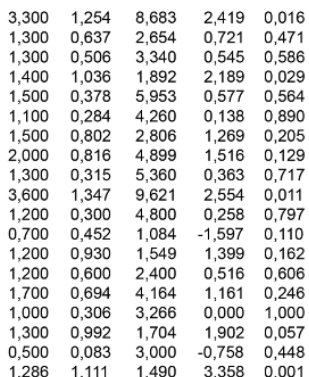

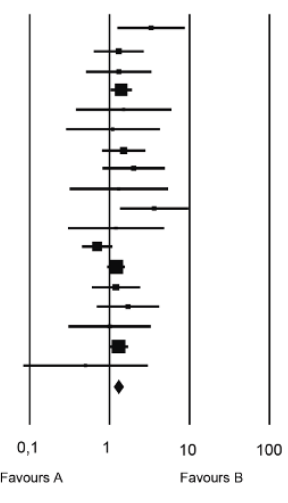

Figure 6 Forest plots depicting risk ratios for familial autoimmunity in probands with AITD and MS. The figure shows two different analyses. From top to bottom: autoimmune thyroid disease (A) and multiple sclerosis (B). The summary effect (random effect model) is depicted as a diamond at the bottom of each analysis. The lateral points of each diamond indicate confidence intervals for this estimate. ${ }^{a}$ Numbers in the study name represent different subgroups within the study. AITD, autoimmune thyroid disease; MS, multiple sclerosis. 


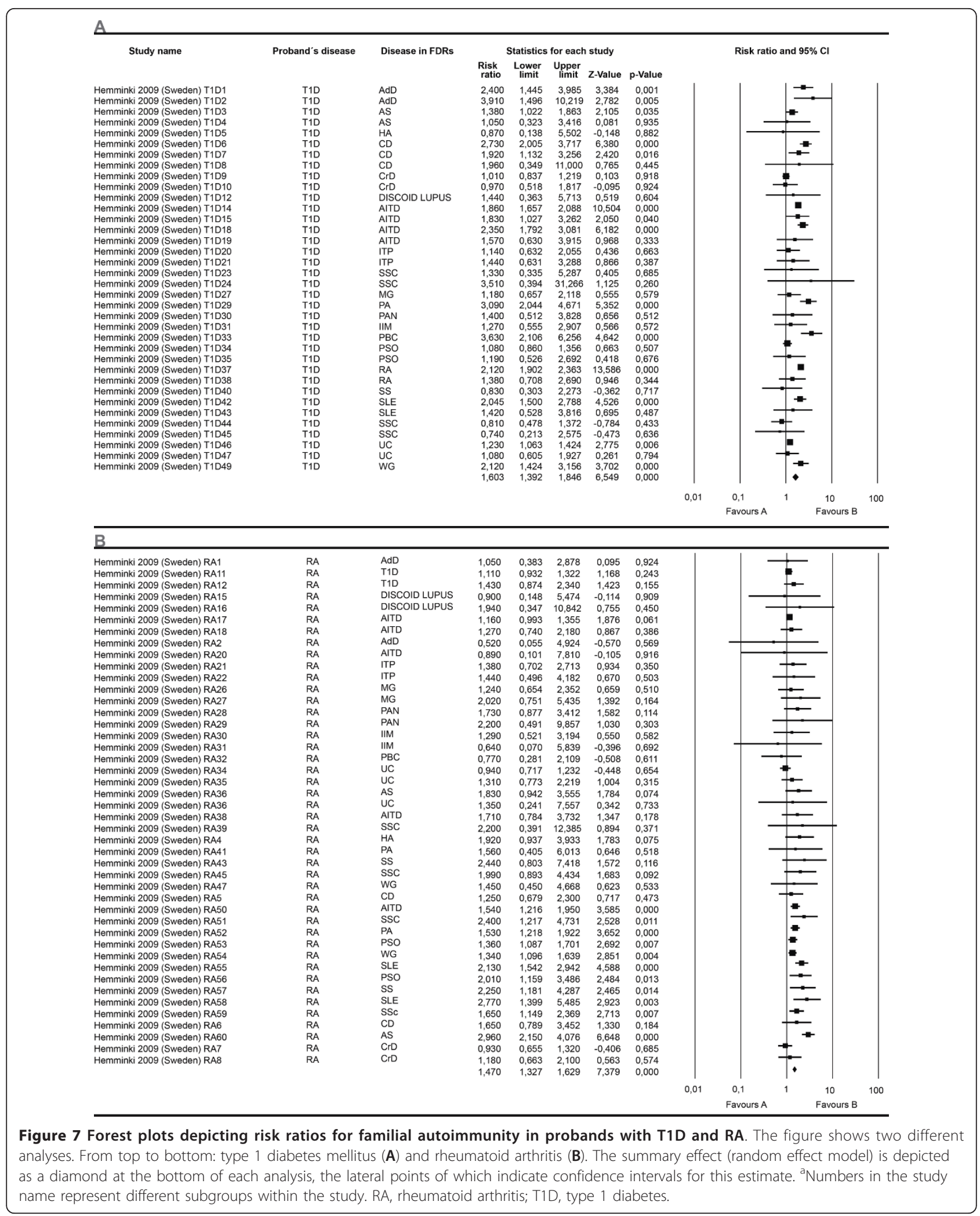


Table 3 Prevalence of specific autoimmune diseases ${ }^{a}$

\begin{tabular}{ll}
\hline Autoimmune disease & Prevalence (\%) \\
\hline Any autoimmune disease & 3 to 8 \\
Addison's disease & 0.009 \\
Alopecia areata & 0.15 \\
Ankylosing spondylitis & 0.20 \\
Autoimmune hepatitis & $\leq 0.001$ \\
Celiac disease & 0.75 \\
CREST syndrome & $\leq 0.001$ \\
Diabetes (type 1) & 0.190 \\
Graves' disease & 1.200 \\
Hashimoto's thyroiditis & 0.800 \\
Idiopathic thrombocytopenic purpura & $\leq 0.001$ \\
Inflammatory bowel disease & 0.450 \\
Juvenile idiopathic arthritis & 0.148 \\
Multiple sclerosis & 0.058 \\
Myasthenia gravis & 0.005 \\
Pernicious anemia & 0.150 \\
Polymyositis/dermatomyositis & 0.005 \\
Primary biliary cirrhosis & 0.004 \\
Psoriasis & 0.75 \\
Rheumatoid arthritis & 1 \\
Sjögren's syndrome & 0.3 \\
Systemic lupus erythematosus & 0.024 \\
Systemic sclerosis (scleroderma) & 0.004 \\
Vitiligo & 0.4 \\
Wegener's granulomatosis & 0.003 \\
\hline PPevances acoring &
\end{tabular}

a Prevalences according to [2,29-32]. CREST, Calcinosis, Raynaud phenomenon, esophageal dysmotility, sclerodactyly, and telangiectasia.

aggregation for diverse ADs, in AITD, T1D, SLE, RA and MS.

\section{Other autoimmune diseases}

The systematic search we performed retrieved other studies that assessed familial autoimmunity besides the five ADs we were focused on. These ADs are SSc, SS, IBD, juvenile dermatomyositis (JDM), VIT, juvenile rheumatoid arthritis (JRA), juvenile SLE (JSLE), idiopathic inflammatory myositis (IIM), CD, and alopecia areata (AA).

\section{Systemic sclerosis}

Four studies reported diagnosis of AITD, RA and SLE in FDRs of SSc probands [57-60]. Frech et al. [60] found a RR of 2.49 (95\% C.I. 1.99 to 3.41) for familial autoimmunity in FDRs and a RR of 1.48 (95\% C.I.1.34 to 2.39) for familial autoimmunity in second degree relatives.

\section{Sjögren's syndrome}

Two studies were found on this disease, one by Reveille et al. [61] and the other done by our group [62]. Both studies agreed on the occurrence of AITD and SLE among relatives. In addition to these ADs, we described the presence of RA [62] while Reveille et al. [61] reported the occurrence of MS and SSc.

\section{Inflammatory bowel disease}

Two studies were retrieved. As mentioned earlier, Criswell et al. [35] found an increased frequency of familial autoimmunity among probands with IBD. A study conducted by Hemminki et al. [63] assessed familial autoimmunity within IBD probands. In UC patients when a parent had AS the SIR for UC in offspring was 1.6, for Crohn's disease (CrD) 2.5, for T1D 1.2, for Graves' disease 1.3, for MS 1.4, for polyarteritis nodosa (PAN) 2.0, for PSO 1.3, for RA 1.1, and for SLE 1.5 [63]. When singleton siblings had CD, the SIR for UC was 2.5, and for AS was 2.1. When a parent and a sibling had CrD the SIR for UC was 4.7 and for PSO was 4.3. In CrD patients, when a parent was diagnosed with UC, the SIR for CrD in offspring was 2.4, for AS was 1.8 and for PSO was 1.4. When singleton siblings had UC, the SIR was 2.8 and for AS was 2.1. When a parent and a sibling had UC, the SIR for CD was 5.0 and for RA was 2.2. In twins, the SIR for CrD-UC pairs was 4.9 [63].

\section{Vitiligo}

For VIT, three studies assessed familial autoimmunity. The studies done by Alkhateeb et al. [64] and Laberge et al. [65] discovered a significant increase in the occurrence of three ADs other than VIT, namely, AITD, PA and AdD. Alkhateeb et al. also reported the occurrence of SLE, myasthenia gravis (MG) and SSc [64], while Laberge et al. found the presence of PSO and RA [65]. In Chinese patients, Zhang et al. [66] found a significant association with RA, AA and PSO.

\section{Juvenile rheumatoid arthritis}

Two studies were found on familial autoimmunity in JRA [67,68]. Prahalad et al. [67] found that AITD accounted for the familial autoimmunity seen in these probands. Huang et al. [68] found, in addition to AITD, the presence of PSO, AS and SLE. Furthermore, Huang et al. [68] compared the prevalence of ADs in family members of probands with JRA against the prevalence in family members of probands with JSLE. Including all family members (that is, first, second and third degree relatives), JSLE probands had a greater prevalence of familial autoimmunity than probands with JRA. Nonetheless, in FDR the prevalence of ADs was not significantly different between these two diseases. Thus, familial autoimmunity is equally present in JRA and JSLE. Likewise, Pachman et al. [89] compared JRA to JDM and to healthy controls. The only statistically significant association was an increased frequency of RA and PA in FDR of JRA probands. 
Table 4 Aggregation of ADs.

\begin{tabular}{|c|c|c|c|c|c|c|c|c|c|c|c|c|c|c|c|c|c|c|c|c|c|c|c|}
\hline \multirow[b]{3}{*}{$A D$ in FDRs } & \multicolumn{23}{|c|}{ Disease in probands } \\
\hline & \multicolumn{5}{|c|}{ T1D } & \multicolumn{2}{|c|}{ AITD } & \multicolumn{3}{|c|}{ SLE } & \multicolumn{4}{|c|}{ RA } & \multicolumn{9}{|c|}{ MS } \\
\hline & $\begin{array}{l}\text { USA } \\
{[35]}\end{array}$ & $\begin{array}{l}\text { UK } \\
\text { [36] }\end{array}$ & $\begin{array}{c}\text { Colombia } \\
\text { [37] }\end{array}$ & $\begin{array}{c}\text { Spain } \\
{[38]}\end{array}$ & $\begin{array}{l}\text { Swed/ } \\
\text { Lith } \\
\text { [40] }\end{array}$ & $\begin{array}{l}\text { UK } \\
{[33]}\end{array}$ & $\begin{array}{l}\text { USA } \\
\text { [35] }\end{array}$ & $\begin{array}{l}\text { USA } \\
{[35]}\end{array}$ & $\begin{array}{l}\text { Latin } \\
\text { America } \\
\text { [42] }\end{array}$ & $\begin{array}{l}\mathrm{NL} \\
{[44]}\end{array}$ & $\begin{array}{c}\text { USA } \\
1 \\
{[35]}\end{array}$ & $\begin{array}{c}\text { USA } \\
2 \\
{[45]}\end{array}$ & $\begin{array}{l}\text { UK } \\
{[46]}\end{array}$ & $\begin{array}{c}\text { USA } \\
3 \\
{[48]}\end{array}$ & $\begin{array}{l}\text { USA1 } \\
\text { [32] }\end{array}$ & $\begin{array}{c}\text { USA } \\
2 \\
{[35]}\end{array}$ & $\begin{array}{c}\text { USA } \\
3 \\
{[87]}\end{array}$ & $\begin{array}{c}\text { Greece } \\
\text { (Multiplex) } \\
{[50]}\end{array}$ & $\begin{array}{c}\text { Greece } \\
\text { (Simplex) } \\
\text { [50] }\end{array}$ & $\begin{array}{c}\text { Australia } \\
\text { [52] }\end{array}$ & $\begin{array}{c}\text { Italy } \\
\text { (Multiplex) } \\
{[53]}\end{array}$ & $\begin{array}{c}\text { Italy } \\
\text { (Simplex) } \\
{[53]}\end{array}$ & $\begin{array}{c}\text { Canada } \\
\text { [54] }\end{array}$ \\
\hline AITD & 11.11 & 0.98 & 2.4 & 4.6 & 0.88 & & & 21.7 & 16 & 20 & 14.7 & 3.9 & 4.81 & 5.50 & & 13,28 & & & & & & & \\
\hline Hyperthyroidism ${ }^{\mathrm{a}}$ & & & & & & 4.2 & & & & & & & & & 3.75 & & 22.1 & 9.42 & 8.2 & 14.15 & & & \\
\hline$\underset{a}{\text { Hypothyroidism }}$ & & & & & & 7.38 & & & & & & & & & & & & 1.71 & 0.74 & 9.43 & & & \\
\hline $\mathrm{T} 1 \mathrm{D}$ & & & 13.5 & & & 7.53 & 78.9 & 39.7 & & 12.53 & 30.9 & 14.49 & & & & 74 & 93.2 & 14.41 & 5.41 & 49.65 & 11.70 & 3.51 & 1.93 \\
\hline RA & & & & 0.9 & & 7.41 & 22.5 & 16.98 & 64 & 28.57 & & & & & 2.0 & 7.8 & 18.7 & 0.68 & 0.18 & 20.75 & & & 1.73 \\
\hline PA & & & 2.14 & & & 12.38 & & & & & & & & & & & & & & & & & \\
\hline SLE & 462 & & 13.35 & & & 5.71 & 312.5 & & & 595 & 163.4 & & & & & 390 & 225 & 42.8 & 25 & 471 & & & \\
\hline $\mathrm{AdD}$ & & & & & & 4.81 & & & & & & & & & & & & & & & & & \\
\hline$C D$ & & & & 3.6 & 0.54 & 0.39 & & & & & & & & & & & & & & & & & \\
\hline VIT & & & 0.8 & 2.75 & & 1.6 & & & & & & & & & & & & 0.86 & 0.09 & 4.72 & & & \\
\hline MS & & & & & & 7.61 & 86.2 & 130 & & 164 & 67.6 & & & & & & & & & & & & \\
\hline MG & & & & & & 9.13 & & & & & & 9.17 & & & & & & 68.49 & 7.09 & & & & \\
\hline IBD & & & & 0.89 & & 1.25 & 30 & 4.2 & & & 21.79 & & & & 4.44 & 24 & & 10.9 & 7.38 & 15.09 & & & \\
\hline PSO & 14.8 & & & 1.6 & & & & 10 & & & 15.69 & & & & 2.67 & 25 & & 5.48 & 1.77 & 33.9 & & & \\
\hline SS & & & & & & & & & & 7.9 & & & & & & & & 6.85 & 1.77 & & & & \\
\hline AS & & & & & & & & & & & & 2.29 & & & & & & & & 56.6 & & & \\
\hline SSC & & & & & & & & & 500 & 595 & & & & & & & & & & 471 & & & \\
\hline$\| \mathrm{M}$ & & & & & & & & & 400 & & & & & & & & & & & & & & \\
\hline
\end{tabular}

Table shows aggregation ( $\lambda$ ) of ADs. The top row depicts the AD present in the proband while the first column shows the AD present in FDRs. The numbers correspond to the calculation of aggregation. ${ }^{\mathrm{a} O n l y}$ autoimmune hypothyroidism and hyperthyroidism are depicted. Prevalences in the general population according to [2,29-32]. If a range was reported the mean was calculated. AdD, Addison's disease; AS, ankylosing spondylitis; AITD, autoimmune thyroid disease; CD, celiac disease; IBD, inflammatory bowel disease; IIM, idiopathic inflammatory myositis; Lith, Lithuania; MG, myasthenia gravis; MS, multiple sclerosis; NL, The Netherlands; PA, pernicious anemia; PSO, psoriasis; RA, rheumatoid arthritis; SLE, systemic lupus erythematosus; SS, Sjögren's syndrome; SSc, systemic sclerosis; Swed, Sweden; T1D, type 1 diabetes; VIT, vitiligo. 


\section{Juvenile systemic lupus erythematosus}

Two articles were found for this disease. While Huang et al. [68] found that 17\% of the FDRs of JSLE probands were affected with an AD, Walters et al. [69] found a prevalence of $51 \%$, with $35 \%$ of FDRs from JSLE probands having SLE, 30\% AITD and 13\% PSO.

\section{Idiopathic inflammatory myositis}

Familial autoimmunity has also been assessed for IIM in two studies. The study by Ginn et al. [70] found that the most common disease was, once again, AITD followed by RA, T1D and PSO. In this article, OR for familial aggregation of ADs was calculated irrespective of disease status (that is, case or control). The strongest predictors were a blood relative and female gender. Niewold et al. [71] reported that FDRs of probands with JDM had a higher frequency of T1D or SLE than in FDRs of controls. However, this relationship did not reach statistical significance.

\section{Celiac disease}

Three articles were found. Petaros et al. [72] found that the prevalence of familial autoimmunity was $4.9 \%$ among first and second degree relatives. The ADs that became manifest were AITD, PSO and T1D. In line with these results, Cataldo et al. [73] found an increased prevalence of ADs including AITD and T1D. Neuhausen et al. [74] also found a significant association with T1D and JRA. However, contrary to what was expected, they found a decreased prevalence of AITD.

\section{Alopecia areata}

An increased frequency of AITD, VIT, T1D, PSO, and CD was found among FDRs of pediatric patients with AA [75].

\section{Discussion}

The results found in this work support aggregation of diverse ADs (that is, familial autoimmunity) and the view of a common origin for ADs (that is, the autoimmune tautology). While polyautoimmunity [7-9,90,91] and familial autoimmune disease $[1,9,42,92-94]$ are well-supported concepts in the medical literature, few articles have familial autoimmunity as their primary concern. Familial autoimmunity is still a topic that has not been thoroughly explored. To our knowledge, this is the first study specifically designed as a systematic review and meta-analysis to find evidence for familial autoimmunity in five major ADs. Familial autoimmunity uses the concept of 'autoimmune disease' as a trait that encompasses all pathologies showing evidence of an autoimmune origin. AITD followed by SLE and RA were the most frequent ADs encountered (Figure 8).

Our meta-analysis was developed in two stages. First, we wanted to determine the presence of familial autoimmunity as a trait in probands with the five index diseases mentioned earlier. However, a meta-analysis of studies having probands with SLE was not feasible. For the other four index diseases the meta-analyses indicate an increased risk of familial autoimmunity with RRs of 2.4, 1.6, 1.5, and 1.3 for AITD, T1D, RA and MS, respectively. It is not surprising to have AITD as the disease with a greater risk for familial autoimmunity as it is the most common AD worldwide. Meta-analyses with ORs as a measure of association were also done showing a significant relationship of familial autoimmunity with RA and MS probands.

Conversely, for our second approach, instead of grouping the studies for the meta-analyses by the proband's disease, we grouped the studies according to the disease

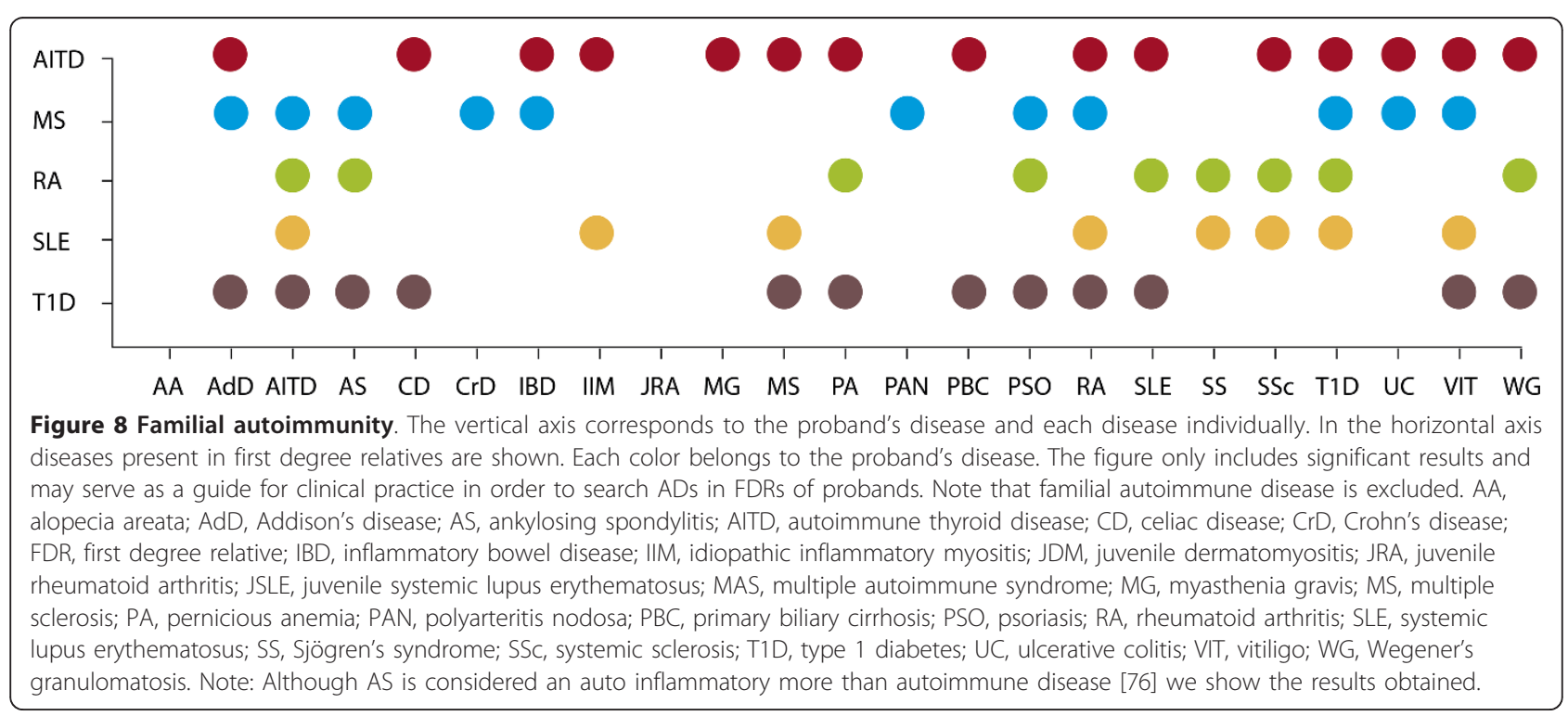


present in FDRs. We must look at familial autoimmunity as a two way relationship depending upon which member of the nuclear family is the proband. Accordingly, we developed our second approach which also disclosed the presence of familial autoimmunity in a variety of ADs (Figure 4 and Additional file 1).

Several reasons may account for the heterogeneity found in our study, which have been also acknowledged by other authors $[90,95]$, namely, different study designs, geographical differences, lack of adequate controls, use of a selected group of probands, and information bias, that is, recall bias [96], diverse population characteristics, and assorted study dates. The quality of studies was certainly influenced by the lack of awareness of familial autoimmunity. In addition, with time diagnostic approaches may have a better performance which may lead to a false increase in diagnoses frequencies.

Aggregation analyses disclosed extreme values (Table 3 and Table 4), with familial recurrence risk values over 100 as in the case of SSc ( $\lambda$ for SSc in FDRs of SLE probands $=500$ to 595 ) or the case for SLE ( $\lambda$ for SLE in FDRs of MS probands $=471$ ). In addition to these extreme values, we had conflicting results as in the case between MS and RA, and T1D and AITD for which some studies found a lack of aggregation whereas others found the opposite. These discrepancies may be explained by the fact that there are differences in prevalence according to geographical location, that aggregation involves genetic and environmental factors and, also, by the arbitrary calculation of means whenever a prevalence range was reported.

In the clinical setting, clinicians should be aware of familial autoimmunity whenever they are attending patients with ADs (Figure 8). A search for autoimmunity in their FDRs should be encouraged by exploring the presence of auto-antibodies [5] and other risk factors $[20,21]$. Since healthy subjects may have positive autoantibody titers, we decided only to include studies that were based on clinical diseases and not on the presence of autoantibodies.

ADs follow a multifactorial (or complex) inheritance pattern which represents an interaction between the collective effect of the genotype at multiple loci (polygenic or multigenic effects) either to raise or to lower susceptibility to disease, combined with a variety of environmental exposures that may trigger, accelerate, exacerbate, or protect against the disease process. Besides assessing the increased frequency of familial autoimmunity, the search also retrieved studies describing how this familial autoimmunity presents. A predominant inheritance of the autoimmunity trait from mothers was evident in some ADs including SS [62], juvenile idiopathic arthritis [97] and T1D [40]. This is indicative of a preferential transmission of susceptibility alleles from mothers to offspring. Maternal transmission of autoimmunity could be influenced by the high preponderance of ADs in women as compared with the general population. However, this higher than expected frequency of maternal transmission of the autoimmunity trait would warrant further studies of mitochondrial DNA, genomic imprinting, maternal-offspring compatibility, gene-environment and indirect genetic effects in ADs [62].

Another factor that influences familial autoimmunity is race $[40,98,99]$. Houghton et al. [98] compared the prevalence of familial autoimmunity between 'native' (Amerindian) and other groups in pediatric patients in the United States. In a small sample (6 Amerindians with SLE versus 34 non Amerindian population with SLE ), $83 \%$ of the native probands had a familial history of ADs while this was true for only $19 \%$ of the non-natives [98]. Meanwhile, with a larger sample size, the GLADEL study found that mestizos had more familial autoimmunity than other racial groups [42]. In fact, ancestry influences the risk and outcome of autoimmunity [99].

We would like to acknowledge the limitations of our study. First, the search was focused on five principal ADs, but we identified articles with probands from other ADs. It is probable that the number of articles retrieved from these ADs is less than if a specific search was done for each of these diseases. Second, we recall the heterogeneity of the study $[100,101]$. Third, in our search we found articles that did not distinguish between the presence of autoantibodies and a clinical diagnosis of an AD. This also should be taken into account in future studies as the presence of autoantibodies may occur in healthy people. Nevertheless, as stated earlier, they may herald a later onset of a given AD and, therefore, it may be clinically important to follow up those individuals.

\section{Conclusions}

The importance of familial autoimmunity has been shown [102]. AITD followed by SLE and RA are the most frequent ADs in familial autoimmunity. Although nongenetic factors may have an effect on familial aggregation, shared genetic factors, in fact, may be the more likely cause for this aggregation [9]. Genes with larger effects (higher penetrance) are related to Mendelian patterns of inheritance, whereas those with smaller effects (lower penetrance) are related rather to complex traits, such as ADs. Identification of such genes, predisposing and affecting the outcome of ADs, is a major challenge for the near future. Given the clinical and etiologic heterogeneity of ADs, understanding the relationship of genotype to phenotype is an extremely important goal for research aimed at gene identification. Thus, further studies of familial autoimmunity will help in increasing the knowledge about 
the common mechanisms of autoimmunity. Genomics and other related disciplines will offer the tools to accomplish this task, allowing us to predict and prevent ADs, tailor individual medical decisions, and provide personalized healthcare while facilitating the patients' participation in their treatment and eventual cure of their disease [103].

\section{Additional material}

Additional file 1: Forest plots depicting risk ratios for familial autoimmunity in first degree relatives. The figure shows two different analyses. From top to bottom: Addison's disease, autoimmune thyroid disease, ankylosing spondylitis, celiac disease, inflammatory bowel disease, discoid lupus, hemolytic anemia, inflammatory idiopathic myositis, immune thrombocytopenic purpura, localized scleroderma, pernicious anemia, myasthenia gravis, multiple sclerosis, polyarteritis nodosa, primary biliary cirrhosis, psoriasis, rheumatoid arthritis, systemic lupus erythematosus, Sjögren's syndrome, systemic sclerosis, type 1 diabetes, vitiligo, Wegener's granulomatosis. The summary effect (random effect model) is depicted as a diamond at the bottom of each analysis. The lateral points of each diamond indicate confidence intervals for this estimate.

Additional file 2: Funnel plots of the three meta-analyses showing publication bias. The corresponding funnel plot shows the standard error on the $Y$ axis and the log value for common effect size on the horizontal axis. From top to bottom: OR for Type 1 diabetes in first degree relatives, OR for multiple sclerosis in probands, RR for autoimmune thyroid disease in probands. Visual inspection of funnel plots suggested effect sizes for the mentioned analyses were scattered asymmetrically around a central effect. OR, odds ratio; RR, risk ratio.

\section{Abbreviations}

AA: alopecia areata; AdD: Addison's disease; AD: autoimmune disease; AITD: autoimmune thyroid disease; AS: ankylosing spondylitis; C\&C: case control; CD: celiac disease; Cl: confidence interval; $C r D$ : Crohn's disease; DL: discoid lupus; FDRs: first degree relatives; GLADEL: Grupo Latinoamericano de Estudio de Lupus; HA: hemolytic anemia; IBD: inflammatory bowel disease; IIM: idiopathic inflammatory myositis; JDM: juvenile dermatomyositis; JRA: juvenile rheumatoid arthritis;JSLE: juvenile systemic lupus erythematosus; MAS: multiple autoimmune syndrome; MG: myasthenia gravis; MS: multiple sclerosis; OR: odds ratio; PA: pernicious anemia; PAN: polyarteritis nodosa; PBC: primary biliary cirrhosis; PSO: psoriasis; RA: rheumatoid arthritis; RR: risk ratio; SIR: standardized incidence ratio; SLE: systemic lupus erythematosus; SS: Sjögren's syndrome; SSc: systemic sclerosis;T1D: type 1 diabetes; UC: ulcerative colitis; VIT: vitiligo; WG: Wegener's granulomatosis; $\lambda$, recurrence risk.

\section{Authors' contributions}

JMA conceived the study, contributed to the literature search and drafted the article. JCR drafted the manuscript, did the literature search and carried out the data analysis. ARV helped to draft the manuscript, was the secondary reviewer for eligibility criteria and carried out the data analysis. All authors read and approved the final version of the manuscript.

\section{Competing interests}

The authors declare that they have no competing interests.

\section{Acknowledgements}

We thank the reviewers for their constructive and helpful suggestions, as well as our colleagues Mauricio Arcos-Burgos, Pierre Youinou and Yehuda Shoenfeld for their fruitful discussions. This work was supported by Universidad del Rosario and Colciencias (122254531722), Bogota, Colombia.

Received: 24 July 2012 Accepted: 18 March 2013 Published: 18 March 2013

\section{References}

1. Anaya JM, Shoenfeld Y, Correa PA, García-Carrasco M, Cervera R Autoinmunidad y Enfermedad Autoinmune Medellín: CIB; 2005.

2. Cooper GS, Stroehla BC: The epidemiology of autoimmune diseases. Autoimmun Rev 2003, 2:119-125.

3. Youinou P, Pers JO, Gershwin ME, Shoenfeld Y: Geo-epidemiology and autoimmunity. J Autoimmun 2010, 34:J163-167.

4. Cho JH, Gregersen PK: Genomics and the multifactorial nature of human autoimmune disease. N Engl I Med 2011, 365:1612-1623.

5. Tobon GJ, Pers JO, Canas CA, Rojas-Villarraga A, Youinou P, Anaya JM: Are autoimmune diseases predictable? Autoimmun Rev 2012, 11:259-266

6. Anaya JM: The autoimmune tautology. Arthritis Res Ther 2010, 12:147.

7. Anaya JM: Common mechanisms of autoimmune diseases (the autoimmune tautology). Autoimmun Rev 2012, 11:781-784.

8. Rojas-Villarraga A, Amaya-Amaya J, Rodriguez-Rodriguez A, Mantilla RD, Anaya JM: Introducing polyautoimmunity: secondary autoimmune diseases no longer exist. Autoimmune Dis 2012, 2012:254319.

9. Anaya JM, Corena R, Castiblanco J, Rojas-Villarraga A, Shoenfeld Y: The kaleidoscope of autoimmunity: multiple autoimmune syndromes and familial autoimmunity. Expert Rev Clin Immunol 2007, 3:623-635.

10. Castiblanco J, Anaya JM: The nature and nurture of common autoimmunity. Ann N Y Acad Sci 2007, 1109:1-8.

11. Lorber M, Gershwin ME, Shoenfeld Y: The coexistence of systemic lupus erythematosus with other autoimmune diseases: the kaleidoscope of autoimmunity. Semin Arthritis Rheum 1994, 24:105-113.

12. Shoenfeld Y: The kaleidoscope of autoimmunity. Autoimmunity 1993, 15:245-252.

13. Sloka S: Observations on recent studies showing increased cooccurrence of autoimmune diseases. J Autoimmun 2002, 18:251-257.

14. Weiss $P$, Shoenfeld $Y$ : Shifts in autoimmune diseases: the kaleidoscope of autoimmunity. Isr J Med Sci 1991, 27:215-217.

15. Humbert P, Dupond JL: Multiple autoimmune syndromes (French). Ann Med Interne (Paris) 1988, 139:159-168.

16. Anaya JM, Castiblanco J, Rojas-Villarraga A, Pineda-Tamayo R, Levy RA, Gomez-Puerta J, Dias C, Mantilla RD, Gallo JE, Cervera R, Shoenfeld Y, ArcosBurgos $\mathrm{M}$ : The multiple autoimmune syndromes. A clue for the autoimmune tautology. Clin Rev Allergy Immunol 2012, 43:256-264.

17. Eisenbarth GS, Gottlieb PA: Autoimmune polyendocrine syndromes. N Engl J Med 2004, 350:2068-2079.

18. Quintero OL, Amador-Patarroyo MJ, Montoya-Ortiz G, Rojas-Villarraga A, Anaya JM: Autoimmune disease and gender: plausible mechanisms for the female predominance of autoimmunity. J Autoimmun 2012, 38 : J109-119.

19. Cruz-Tapias P, Perez-Fernandez OM, Rojas-Villarraga A, RodriguezRodriguez A, Arango MT, Anaya JM: Shared HLA class II in six autoimmune diseases in Latin America: A meta-analysis. Autoimmune Dis 2012, 2012:569728.

20. Barragan-Martinez C, Speck-Hernandez CA, Montoya-Ortiz G, Mantilla RD, Anaya JM, Rojas-Villarraga A: Organic solvents as risk factor for autoimmune diseases: a systematic review and meta-analysis. PLoS One 2012, 7:e51506.

21. George J, Levy Y, Shoenfeld Y: Smoking and immunity: an additional player in the mosaic of autoimmunity. Scand J Immunol 1997, 45:1-6.

22. Arbuckle MR, McClain MT, Rubertone MV, Scofield RH, Dennis GJ, James JA, Harley JB: Development of autoantibodies before the clinical onset of systemic lupus erythematosus. N Engl J Med 2003, 349:1526-1533.

23. Scofield RH: Autoantibodies as predictors of disease. Lancet 2004, 363:1544-1546.

24. Harel M, Shoenfeld Y: Predicting and preventing autoimmunity, myth or reality? Ann N Y Acad Sci 2006, 1069:322-345.

25. Shepshelovich D, Shoenfeld Y: Prediction and prevention of autoimmune diseases: additional aspects of the mosaic of autoimmunity. Lupus 2006, 15:183-190.

26. Sherer Y, Shoenfeld Y: Antiphospholipid antibodies: are they proatherogenic or an epiphenomenon of atherosclerosis? Immunobiology 2003, 207:13-16.

27. Shoenfeld Y: Primary biliary cirrhosis and autoimmune rheumatic diseases: prediction and prevention. Isr J Med Sci 1992, 28:113-116.

28. OCEBM Levels of Evidence Working Group*: "The Oxford 2011 levels of evidence". Oxford Centre for Evidence-Based Medicine [http://www.cebm.net/ index.aspx?o $=5653]$. 
29. Madani S, Shapiro J: Alopecia areata update. J Am Acad Dermatol 2000 42:549-566.

30. Cotch MF, Hoffman GS, Yerg DE, Kaufman Gl, Targonski P, Kaslow RA: The epidemiology of Wegener's granulomatosis. Estimates of the five-year period prevalence, annual mortality, and geographic disease distribution from population-based data sources. Arthritis Rheum 1996, 39:87-92.

31. Jacobson DL, Gange SJ, Rose NR, Graham NM: Epidemiology and estimated population burden of selected autoimmune diseases in the United States. Clin Immunol Immunopathol 1997, 84:223-243.

32. Barcellos LF, Kamdar BB, Ramsay PP, DeLoa C, Lincoln RR, Caillier S, Schmidt S, Haines JL, Pericak-Vance MA, Oksenberg JR, Hauser SL: Clustering of autoimmune diseases in families with a high-risk for multiple sclerosis: a descriptive study. Lancet Neurol 2006, 5:924-931.

33. Boelaert K, Newby PR, Simmonds MJ, Holder RL, Carr-Smith JD, Heward JM, Manji N, Allahabadia A, Armitage M, Chatterjee KV, Lazarus JH, Pearce SH, Vaidya B, Gough SC, Franklyn JA: Prevalence and relative risk of other autoimmune diseases in subjects with autoimmune thyroid disease. Am J Med 2010, 123:183.

34. Hemminki K, Li X, Sundquist J, Sundquist K: The epidemiology of Graves' disease: evidence of a genetic and an environmental contribution. J Autoimmun 2010, 34:J307-J313.

35. Criswell LA, Pfeiffer KA, Lum RF, Gonzales B, Novitzke J, Kern M, Moser KL, Begovich AB, Carlton VE, Li W, Lee AT, Ortmann W, Behrens TW, Gregersen PK: Analysis of families in the multiple autoimmune disease genetics consortium (MADGC) collection: the PTPN22 620W allele associates with multiple autoimmune phenotypes. Am J Hum Genet 2005, 76:561-571.

36. Bottazzo GF, Mann Jl, Thorogood M: Autoimmunity in juvenile diabetics and their families. BMJ 1978, 2:165-168.

37. Anaya JM, Castiblanco J, Tobon GJ, Garcia J, Abad V, Cuervo H, Velasquez A, Angel ID, Vega P, Arango A: Familial clustering of autoimmune diseases in patients with type 1 diabetes mellitus. J Autoimmun 2006, 26:208-214

38. Wagner AM, Santana A, Hernndez M, Wiebe JC, Novoa J, Mauricio D: Predictors of associated autoimmune diseases in families with type 1 diabetes: results from the Type 1 Diabetes Genetics Consortium. Diabetes Metab Res Rev 2011, 27:493-498.

39. Hemminki K, Li X, Sundquist J, Sundquist K: Familial association between type 1 diabetes and other autoimmune and related diseases. Diabetologia 2009, 52:1820-1828.

40. Samuelsson U, Sadauskaite V, Padaiga Z, Ludvigsson J: A fourfold difference in the incidence of type 1 diabetes between Sweden and Lithuania but similar prevalence of autoimmunity. Diabetes Res Clin Pract 2004, 66:173-181.

41. Lebenthal Y, Yackobovitch-Gavan M, de Vries L, Phillip M, Lazar L: Coexistent autoimmunity in familial type 1 diabetes: increased susceptibility in sib-pairs? Horm Res Paediatr 2011, 75:284-290.

42. Alarcon-Segovia D, Alarcon-Riquelme ME, Cardiel MH, Caeiro F, Massardo L, Villa AR, Pons-Estel BA: Familial aggregation of systemic lupus erythematosus, rheumatoid arthritis, and other autoimmune diseases in 1,177 lupus patients from the GLADEL cohort. Arthritis Rheum 2005, 52:1138-1147.

43. Priori R, Medda E, Conti F, Cassara EAM, Danieli MG, Gerli R, Giacomelli R, Franceschini F, Manfredi A, Pietrogrande M, Stazi MA, Valesini G: Familial autoimmunity as a risk factor for systemic lupus erythematosus and vice versa: a case-control study. Lupus 2003, 12:735-740.

44. Corporaal S, Bijl M, Kallenberg CG: Familial occurrence of autoimmune diseases and autoantibodies in a Caucasian population of patients with systemic lupus erythematosus. Clin Rheumatol 2002, 21:108-113.

45. Lin JP, Cash JM, Doyle SZ, Peden S, Kanik K, Amos Cl, Bale SJ, Wilder RL: Familial clustering of rheumatoid arthritis with other autoimmune diseases. Hum Genet 1998, 103:475-482.

46. Thomas DJ, Young A, Gorsuch AN, Bottazzo GF, Cudworth AG: Evidence for an association between rheumatoid arthritis and autoimmune endocrine disease. Ann Rheum Dis 1983, 42:297-300.

47. Hemminki K, Li X, Sundquist J, Sundquist K: Familial associations of rheumatoid arthritis with autoimmune diseases and related conditions. Arthritis Rheum 2009, 60:661-668.

48. Jawaheer D, Lum RF, Amos Cl, Gregersen PK, Criswell LA: Clustering of disease features within 512 multicase rheumatoid arthritis families. Arthritis Rheum 2004, 50:736-741.
49. Broadley SA, Deans J, Sawcer SJ, Clayton D, Compston DA: Autoimmune disease in first-degree relatives of patients with multiple sclerosis. A UK survey. Brain 2000, 123:1102-1111.

50. Deretzi G, Kountouras J, Koutlas E, Zavos C, Polyzos S, Rudolf J, Grigoriadis N, Gavalas E, Boziki M, Tsiptsios I: Familial prevalence of autoimmune disorders in multiple sclerosis in Northern Greece. Mult Scler 2010, 16:1091-1101.

51. Heinzlef O, Alamowitch S, Sazdovitch V, Chillet P, Joutel A, TournierLasserve $E$, Roullet E: Autoimmune diseases in families of French patients with multiple sclerosis. Acta Neurol Scand 2000, 101:36-40.

52. Henderson RD, Bain CJ, Pender MP: The occurrence of autoimmune diseases in patients with multiple sclerosis and their families. J Clin Neurosci 2000, 7:434-437.

53. Marrosu MG, Cocco E, Lai M, Spinicci G, Pischedda MP, Contu P: Patients with multiple sclerosis and risk of type 1 diabetes mellitus in Sardinia, Italy: a cohort study. Lancet 2002, 359:1461-1465.

54. Ramagopalan SV, Dyment DA, Valdar W, Herrera BM, Criscuoli M, Yee IM, Sadovnick AD, Ebers GC: Autoimmune disease in families with multiple sclerosis: a population-based study. Lancet Neurol 2007, 6:604-610.

55. Nielsen NM, Frisch M, Rostgaard K, Wohlfahrt J, Hjalgrim H, KochHenriksen N, Melbye M, Westergaard T: Autoimmune diseases in patients with multiple sclerosis and their first-degree relatives: a nationwide cohort study in Denmark. Mult Scler 2008, 14:823-829.

56. Laroni A, Calabrese M, Perini P, Albergoni MP, Ranzato F, Tiberio M, Battistin L, Gallo P: Multiple sclerosis and autoimmune diseases: epidemiology and HLA-DR association in North-east Italy. J Neurol 2006, 253:636-639.

57. Arora-Singh RK, Assassi S, del Junco DJ, Arnett FC, Perry M, Irfan U, Sharif R, Mattar T, Mayes MD: Autoimmune diseases and autoantibodies in the first degree relatives of patients with systemic sclerosis. J Autoimmun 2010, 35:52-57.

58. Hudson M, Rojas-Villarraga A, Coral-Alvarado P, Lopez-Guzman S, Mantilla RD, Chalem P, Baron M, Anaya JM: Polyautoimmunity and familial autoimmunity in systemic sclerosis. J Autoimmun 2008, 31:156-159.

59. Koumakis E, Dieudé P, Avouac J, Kahan A, Allanore Y: Familial autoimmunity in systemic sclerosis - Results of a French-based casecontrol family study. J Rheumatol 2012, 39:532-538.

60. Frech T, Khanna D, Markewitz B, Mineau G, Pimentel R, Sawitzke A: Heritability of vasculopathy, autoimmune disease, and fibrosis in systemic sclerosis: a population-based study. Arthritis Rheum 2010, 62:2109-2116.

61. Reveille JD, Wilson RW, Provost TT, Bias WB, Arnett FC: Primary Sjogren's syndrome and other autoimmune diseases in families. Prevalence and immunogenetic studies in six kindreds. Ann Intern Med 1984, 101:748-756.

62. Anaya JM, Tobon GJ, Vega P, Castiblanco J: Autoimmune disease aggregation in families with primary Sjogren's syndrome. J Rheumatol 2006, 33:2227-2234.

63. Hemminki K, Li X, Sundquist K, Sundquist J: Familial association of inflammatory bowel diseases with other autoimmune and related diseases. Am J Gastroenterol 2010, 105:139-147.

64. Alkhateeb A, Fain PR, Thody A, Bennett DC, Spritz RA: Epidemiology of vitiligo and associated autoimmune diseases in Caucasian probands and their families. Pigment Cell Res 2003, 16:208-214.

65. Laberge G, Mailloux CM, Gowan K, Holland P, Bennett DC, Fain PR, Spritz RA: Early disease onset and increased risk of other autoimmune diseases in familial generalized vitiligo. Pigment Cell Res 2005, 18:300-305.

66. Zhang Z, Xu SX, Zhang FY, Yin XY, Yang S, Xiao FL, Du WH, Wang JF, LV YM, Tang HY, Zhang XJ: The analysis of genetics and associated autoimmune diseases in Chinese vitiligo patients. Arch Dermatol Res 2009, 301:167-173.

67. Prahalad S, Shear ES, Thompson SD, Giannini EH, Glass DN: Increased prevalence of familial autoimmunity in simplex and multiplex families with juvenile rheumatoid arthritis. Arthritis Rheum 2002, 46:1851-1856.

68. Huang CM, Yang YH, Chiang BL: Different familial association patterns of autoimmune diseases between juvenile-onset systemic lupus erythematosus and juvenile rheumatoid arthritis. I Microbiol Immunol Infect 2004, 37:88-94.

69. Walters HM, Pan N, Moorthy LN, Ward MJ, Peterson MG, Lehman TJ: Patterns and influence of familial autoimmunity in pediatric systemic lupus erythematosus. Pediatr Rheumatol Online J 2012, 10:22. 
70. Ginn LR, Lin JP, Plotz PH, Bale SJ, Wilder RL, Mbauya A, Miller FW: Familial autoimmunity in pedigrees of idiopathic inflammatory myopathy patients suggests common genetic risk factors for many autoimmune diseases. Arthritis Rheum 1998, 41:400-405.

71. Niewold TB, Wu SC, Smith M, Morgan GA, Pachman LM: Familial aggregation of autoimmune disease in juvenile dermatomyositis. Pediatrics 2011, 127:e1239-1246.

72. Petaros P, Martelossi S, Tommasini A, Torre G, Caradonna M, Ventura A: Prevalence of autoimmune disorders in relatives of patients with celiac disease. Dig Dis Sci 2002, 47:1427-1431.

73. Cataldo F, Marino V: Increased prevalence of autoimmune diseases in first-degree relatives of patients with celiac disease. J Pediatr Gastroenterol Nutr 2003, 36:470-473.

74. Neuhausen SL, Steele L, Ryan S, Mousavi M, Pinto M, Osann KE, Flodman P, Zone JJ: Co-occurrence of celiac disease and other autoimmune diseases in celiacs and their first-degree relatives. J Autoimmun 2008, 31:160-165.

75. Kakourou T, Karachristou K, Chrousos G: A case series of alopecia areata in children: impact of personal and family history of stress and autoimmunity. J Eur Acad Dermatol Venereol 2007, 21:356-359.

76. Pérez-Fernández OM, Mantilla RD, Cruz-Tapias P, Rodriguez-Rodriguez A, Rojas-Villarraga A, Anaya J-M: Spondyloarthropathies in autoimmune diseases and vice versa. Autoimmune Dis 2012, 2012:736384.

77. Roberts CGP, Ladenson PW: Hypothyroidism. Lancet 2004, 363:793-803.

78. Shapira Y, Agmon-Levin N, Shoenfeld Y: Defining and analyzing geoepidemiology and human autoimmunity. J Autoimmun 2010, 34 J168-177.

79. Wiebolt J, Achterbergh $\mathrm{R}$, den Boer $\mathrm{A}$, van der Leij S, Marsch E, Suelmann B, de Vries R, van Haeften TW: Clustering of additional autoimmunity behaves differently in Hashimoto's patients compared with Graves' patients. Eur J Endocrinol 2011, 164:789-794.

80. Szyper-Kravitz M, Marai I, Shoenfeld Y: Coexistence of thyroid autoimmunity with other autoimmune diseases: friend or foe? Additional aspects on the mosaic of autoimmunity. Autoimmunity 2005, 38:247-255.

81. Dittmar M, Libich C, Brenzel T, Kahaly GJ: Increased familial clustering of autoimmune thyroid diseases. Horm Metab Res 2011, 43:200-204.

82. Scofield RH, Bruner GR, Harley JB, Namjou B: Autoimmune thyroid disease is associated with a diagnosis of secondary Sjogren's syndrome in familial systemic lupus. Ann Rheum Dis 2007, 66:410-413.

83. Taneja V, Singh RR, Malaviya AN, Anand C, Mehra NK: Occurrence of autoimmune diseases and relationship of autoantibody expression with HLA phenotypes in multicase rheumatoid arthritis families. Scand J Rheumatol 1993, 22:152-157.

84. Walker DJ, Griffiths M, Griffiths ID: Occurrence of autoimmune diseases and autoantibodies in multicase rheumatoid arthritis families. Ann Rheum Dis 1986, 45:323-326.

85. Midgard R, Gronning M, Riise T, Kvale G, Nyland H: Multiple sclerosis and chronic inflammatory diseases. A case-control study. Acta Neurol Scand 1996, 93:322-328.

86. Annunziata P, Morana P, Giorgio A, Galeazzi M, Campanella V, Lore F, Guarino E: High frequency of psoriasis in relatives is associated with early onset in an Italian multiple sclerosis cohort. Acta Neurol Scand 2003, 108:327-331.

87. Alonso A, Hernan MA, Ascherio A: Allergy, family history of autoimmune diseases, and the risk of multiple sclerosis. Acta Neurol Scand 2008, 117:15-20.

88. Magana-Zamora L, Chiquete E, Campos-Gonzalez ID, Cantu-Leal R, IbarraBravo O, Punzo-Bravo G, Rojas-Flores I: Risk factors and outcome of patients with multiple sclerosis from the state of Michoacan Mexico: a case-control study. Rev Mex Neuroci 2012, 13:78-85.

89. Pachman LM, Hayford JR, Hochberg MC, Pallansch MA, Chung A, Daugherty CD, Athreya BH, Bowyer SL, Fink CW, Gewanter HL, Jerath R, Lang BA, Szer IS, Sinacore J, Christensen ML, Dyer AR: New-onset juvenile dermatomyositis: comparisons with a healthy cohort and children with juvenile rheumatoid arthritis. Arthritis Rheum 1997, 40:1526-1533.

90. Somers EC, Thomas SL, Smeeth L, Hall AJ: Autoimmune diseases cooccurring within individuals and within families: a systematic review. Epidemiology 2006, 17:202-217.

91. Cárdenas Roldán J, Amaya-Amaya J, Castellanos-de la Hoz J, GiraldoVillamil J, Montoya-Ortiz G, Cruz-Tapias P, Rojas-Villarraga A, Mantilla RD,
Anaya J-M: Autoimmune thyroid disease in rheumatoid arthritis: a global perspective. Arthritis 2012, 2012:864907.

92. Harjutsalo V, Podar T, Tuomilehto J: Cumulative incidence of type 1 diabetes in 10,168 siblings of Finnish young-onset type 1 diabetic patients. Diabetes 2005, 54:563-569.

93. Brix TH, Kyvik KO, Hegedus L: A population-based study of chronic autoimmune hypothyroidism in Danish twins. J Clin Endocrinol Metab 2000, 85:536-539.

94. Deighton CM, Walker DJ: The familial nature of rheumatoid arthritis. Ann Rheum Dis 1991, 50:62-65.

95. Eaton WW, Rose NR, Kalaydjian A, Pedersen MG, Mortensen PB: Epidemiology of autoimmune diseases in Denmark. J Autoimmun 2007, 29:1-9.

96. Marrie RA: Autoimmune disease and multiple sclerosis: methods, methods, methods. Lancet Neurol 2007, 6:575-576.

97. Zeft A, Shear ES, Thompson SD, Glass DN, Prahalad S: Familial autoimmunity: maternal parent-of-origin effect in juvenile idiopathic arthritis. Clin Rheumatol 2008, 27:241-244.

98. Houghton KM, Page J, Cabral DA, Petty RE, Tucker LB: Systemic lupus erythematosus in the pediatric North American Native population of British Columbia. J Rheumatol 2006, 33:161-163.

99. Sanchez E, Rasmussen A, Riba L, Acevedo E, Kelly JA, Langefeld CD, GarciaDe La Torre I, Maradiaga-Cecena MA, Cardiel MH, Esquivel-Valerio JA, Rodriguez-Amado J, Moctezuma JF, Miranda P, Perandones C, Castel C, Laborde HA, Alba P, Musuruana J, Goecke A, Anaya JM, Kaufman KM, Adler A, Brown EE, Alarcon GS, Kimberly RP, Edberg JC, Criswell LA, Gilkeson GS, Niewold TB, Martin J, et al: Impact of genetic ancestry and socio-demographic status on the clinical expression of systemic lupus erythematosus in Amerindian-European populations. Arthritis Rheum 2012, 64:3687-3694.

100. Liberati A, Altman DG, Tetzlaff J, Mulrow C, Gøtzsche PC, loannidis JPA, Clarke M, Devereaux PJ, Kleijnen J, Moher D: The PRISMA statement for reporting systematic reviews and meta-analyses of studies that evaluate health care interventions: explanation and elaboration. J Clin Epidemiol 2009, 62:e1-34

101. Stroup DF, Berlin JA, Morton SC, Olkin I, Williamson GD, Rennie D, Moher D, Becker BJ, Sipe TA, Thacker SB: Meta-analysis of observational studies in epidemiology: a proposal for reporting. Meta-analysis Of Observational Studies in Epidemiology (MOOSE) group. JAMA 2000, 283:2008-2012.

102. Anaya JM, Shoenfeld Y, Cervera R: Facts and challenges for the autoimmunologist. Lessons from the second Colombian autoimmune symposium. Autoimmun Rev 2012, 11:249-251.

103. Anaya JM, Rojas-Villarraga A, Garcia-Carrasco M: The autoimmune tautology: from polyautoimmunity and familial autoimmunity to the autoimmune genes. Autoimmune Dis 2012, 2012:297193.

\section{Pre-publication history}

The pre-publication history for this paper can be accessed here: http://www.biomedcentral.com/1741-7015/11/73/prepub

doi:10.1186/1741-7015-11-73

Cite this article as: Cárdenas-Roldán et al:: How do autoimmune diseases cluster in families? A systematic review and meta-analysis. BMC Medicine 2013 11:73.

\section{Submit your next manuscript to BioMed Central and take full advantage of:}

- Convenient online submission

- Thorough peer review

- No space constraints or color figure charges

- Immediate publication on acceptance

- Inclusion in PubMed, CAS, Scopus and Google Scholar

- Research which is freely available for redistribution

Submit your manuscript at www.biomedcentral.com/submit
C Biomed Central 